TRANSACTIONS OF THE

AMERICAN MATHEMATICAL SOCIETY

Volume 366, Number 4, April 2014, Pages 1905-1941

S 0002-9947(2013)05905-3

Article electronically published on September 4, 2013

\title{
SINGULAR NEUMANN PROBLEMS AND LARGE-TIME BEHAVIOR OF SOLUTIONS OF NONCOERCIVE HAMILTON-JACOBI EQUATIONS
}

\author{
YOSHIKAZU GIGA, QING LIU, AND HIROYOSHI MITAKE
}

\begin{abstract}
We investigate the large-time behavior of viscosity solutions of Hamilton-Jacobi equations with noncoercive Hamiltonian in a multidimensional Euclidean space. Our motivation comes from a model describing growing faceted crystals recently discussed by E. Yokoyama, Y. Giga and P. Rybka. Surprisingly, growth rates of viscosity solutions of these equations depend on the $x$-variable. In a part of the space called the effective domain, growth rates are constant, but outside of this domain, they seem to be unstable. Moreover, on the boundary of the effective domain, the gradient with respect to the $x$-variable of solutions blows up as time goes to infinity. Therefore, we are naturally led to study singular Neumann problems for stationary HamiltonJacobi equations. We establish the existence, stability and comparison results for singular Neumann problems and apply the results for a large-time asymptotic profile on the effective domain of viscosity solutions of Hamilton-Jacobi equations with noncoercive Hamiltonian.
\end{abstract}

\section{Contents}

1. Introduction

1.1. Large-time behavior of solutions

1.2. Singular Neumann problems

1.3. Physical background

2. A basic example in one-dimension

3. Singular Neumann problems

3.1. Definition, existence and stability

3.2. Comparison result

4. Large-time behavior of solutions

4.1. Large-time asymptotics

4.2. Construction of subsolutions and supersolutions of (C)

4.3. Stability and Proof of Theorem 4.1

4.4. Asymptotic profile on the effective domain $\boldsymbol{\Omega}_{\mathbf{e}}$

Received by the editors October 22, 2010 and, in revised form, June 7, 2012.

2010 Mathematics Subject Classification. Primary 35B40, 35F25, 35F30, 49L25.

Key words and phrases. Large-time behavior, noncoercive Hamilton-Jacobi equation, effective domain, singular Neumann problems, gradient grow-up, faceted crystals, facet instability, viscosity solution.

The work of the first author was partly supported by Grant-in-Aid for Scientific Research, No. 21224001 (Kiban S) and No 23244015 (Kiban A), the Japan Society for the Promotion of Science (JSPS).

The work of the second author was partly supported by Research Fellowship for Young Researcher from JSPS, No. 21-7428.

The work of the third author was partly supported by Research Fellowship for Young Researcher from JSPS, No. 22-1725. 
4.5. A model describing growing faceted crystals

5. Appendix

5.1. Explanation for a derivation of (1.1)

5.2. Another characterization of solutions of $(\mathrm{S})$

5.3. Uniform continuity of subsolutions for $(\mathrm{N})$

5.4. Lipschitz estimates and derivative bounds

\section{INTRODUCTION}

In this paper we consider the Cauchy problem for Hamilton-Jacobi (HJ) equations

$$
\begin{cases}u_{t}+H(x, D u)=f(x) & \text { in } \mathbb{R}^{N} \times(0, \infty), \\ u(\cdot, 0)=u_{0} & \text { in } \mathbb{R}^{N},\end{cases}
$$

with noncoercive Hamiltonian $H: \mathbb{R}^{N} \times \mathbb{R}^{N} \rightarrow \mathbb{R}$ of the form

$$
H(x, p)=\sigma(x) m(\|p\|) .
$$

Here $\|\cdot\|$ denotes an arbitrary norm in $\mathbb{R}^{N}$ while $\sigma, f: \mathbb{R}^{N} \rightarrow[0, \infty)$ and $m$ : $[0, \infty) \rightarrow[0,1)$ are continuous functions. Moreover $m(r)$ is assumed to be

$$
\text { strictly increasing and } m(r) \rightarrow 1 \text { as } r \rightarrow \infty \text {. }
$$

The functions $\sigma, f$ and $m$ are given functions. The function $u: \mathbb{R}^{N} \times[0, \infty) \rightarrow \mathbb{R}$ is an unknown function while $u_{0}: \mathbb{R}^{N} \rightarrow \mathbb{R}$ is a given initial value which is assumed to be Lipschitz continuous. To be consistent with the theory of crystal growth [44] we call $\sigma, f$ and $m$ a surface supersaturation, an external force at point $x$ and a kinetic coefficient, respectively. Throughout the paper, we denote $u_{t}:=\partial u / \partial t$ and $D u:=\left(\partial u / \partial x_{1}, \ldots, \partial u / \partial x_{n}\right)$.

A very primitive example which we have in mind is

$$
\begin{cases}u_{t}+\frac{2}{\pi} \arctan \left(u_{x}^{2}\right)=|x| & \text { in } \mathbb{R} \times(0, \infty), \\ u(x, 0)=0 & \text { for all } x \in \mathbb{R} .\end{cases}
$$

If $u$ is a solution of the one-dimensional Cauchy problem, then the large-time asymptotic behavior of $u$ can be described by

$$
u(\cdot, t) \rightarrow u_{\infty} \text { uniformly on }[-1,1]
$$

where

and

$$
u_{\infty}(x)=\int_{0}^{|x|}\left(\tan \left(\frac{\pi}{2} y\right)\right)^{1 / 2} d y \text { for all } x \in[-1,1]
$$

$$
u(\cdot, t) \rightarrow+\infty \text { uniformly on each compact subset of }(-\infty,-1) \cup(1, \infty)
$$

as $t \rightarrow \infty$. This large-time behavior is easily obtained by the method of characteristics and we give a more detailed explanation in Section 2. From this example we learn that the growth rate of $u$ may depend on the $x$-variable explicitly. We emphasize that this phenomenon seems to be new, at least from the viewpoint of study for the large-time behavior of solutions of HJ equations. The typical result 
of study for this asymptotic problem for HJ equations with (coercive) Hamiltonian, which will be explained more precisely later, shows that solutions converge (locally) uniformly with the constant growth rate in the whole domain which is considered as time goes to infinity.

Roughly speaking, on the one hand, the viscosity solution of (C) has the constant growth rate asymptotically in a subset in the whole domain $\mathbb{R}^{N}$, which we will call the effective domain for $(\mathrm{C})$, and on the other hand, outside of the effective domain, the viscosity solution of (C) has an unstable growth rate. In 44, an effective domain is called a maximal stable region of a growing facet. The other feature to be noted is that gradient grow-up (or infinite time gradient blow-up) of solutions happens. More precisely, let $u$ be the solution of $(\mathrm{C}), \Omega_{e} \subset \mathbb{R}^{N}$ be the effective domain and $c$ be the growth rate on $\Omega_{e}$, and then the normal derivative with respect to the $x$-variable of $u-c t$ blows up on the boundary $\partial \Omega_{e}$ of $\Omega_{e}$ as time goes to infinity, i.e.,

$$
D(u(x, t)-c t) \cdot n(x) \rightarrow+\infty \text { for all } x \in \partial \Omega_{e} \text { as } t \rightarrow \infty,
$$

whereas $u-c t$ remains bounded on $\bar{\Omega}_{e}=\Omega_{e} \cup \partial \Omega_{e}$. We refer to [40, 43] and the references therein for results on gradient blow-up and grow-up of solutions of parabolic equations.

One of the aims of this paper is to investigate the large-time behavior of viscosity solutions of $(\mathrm{C})$. More precisely, we give the formulas of the effective domain and the growth rate and prove that viscosity solutions of $(\mathrm{C})$ converge uniformly on the effective domain and that outside of the effective domain they have growth rates which are higher than that of the effective domain.

It turns out that the asymptotic profile on the effective domain is reduced to stationary problems. However, we encounter a difficulty related to the boundary value problem for stationary HJ equations. More precisely, we are led to consider the singular Neumann problem for stationary HJ equations:

$$
\begin{cases}F(x, D u)=h(x) & \text { in } \Omega, \\ \frac{\partial u}{\partial n}=+\infty & \text { on } \partial \Omega, \\ \sup _{x \in \Omega}|u(x)|<+\infty, & \end{cases}
$$

where $\Omega$ is a bounded domain in $\mathbb{R}^{N}, n(x)$ is the outer unit normal vector at $x$ to $\partial \Omega, F: \bar{\Omega} \times \mathbb{R}^{N} \rightarrow \mathbb{R}$ and $h: \Omega \rightarrow[0, \infty)$ are given continuous functions which satisfy $F(x, p) \geq F(x, 0)=0$ for any $(x, p) \in \bar{\Omega} \times \mathbb{R}^{N}, F(x, p)$ is convex and $F$ is coercive with respect to the $p$-variable, i.e.,

$$
F\left(x, \lambda p_{1}+(1-\lambda) p_{2}\right) \leq \lambda F\left(x, p_{1}\right)+(1-\lambda) F\left(x, p_{2}\right)
$$

for any $x \in \bar{\Omega}, p_{1}, p_{2} \in \mathbb{R}^{N}$ and $\lambda \in[0,1]$,

$$
\inf \{F(x, p)|x \in \bar{\Omega},| p \mid \geq r\} \rightarrow+\infty \text { as } r \rightarrow \infty
$$

and

$$
h(x) \rightarrow+\infty \quad \text { as dist }(x, \partial \Omega) \rightarrow 0 \text { and }\{x \in \Omega \mid h(x)=0\} \neq \emptyset,
$$

where $\operatorname{dist}(x, \partial \Omega):=\min \{|x-y| \mid y \in \partial \Omega\}$ for all $x \in \bar{\Omega}$. We call $u$ a solution of (N) if $u$ is a bounded viscosity solution of (1.5) and satisfies that

$$
u-\phi \text { never has a local minimum on } \bar{\Omega}
$$

$$
\text { at the boundary } \partial \Omega \text { for any } \phi \in C^{1}(\bar{\Omega}) \text {. }
$$


This definition was introduced by J.-M. Lasry and P.-L. Lions in 34. In order to distinguish a viscosity solution of (1.5) which blows up at some points on the boundary, i.e., $u(x) \rightarrow+\infty$ as $x \rightarrow x_{0} \in \partial \Omega$, we impose (1.7), since it is clear that blow-up solutions satisfy (1.8). In fact, notice that in the example above, we have

$$
\left.\left(u_{\infty}\right)_{x}(x)\right|_{x= \pm 1}= \pm \infty \text {. }
$$

In the first part of this paper, we establish the existence and stability results for $(\mathrm{N})$ under the additional Assumption (A7), which is a kind of growth condition on $h$. We also prove that the comparison principle holds under the additional Assumption (A9), which is a convexity of sublevel sets of $h$ when $F(x, p)=\|p\|$. See Section 3 for the details of (A7) and (A9). In the latter part of this paper we use these results for $(\mathrm{N})$ for the study of the large-time behavior of viscosity solutions of (C).

1.1. Large-time behavior of solutions. In the last decade, a lot of works have been devoted to the study of large-time behavior of viscosity solutions of HJ equations

$$
u_{t}+H(x, D u)=0 \quad \text { in } \mathbb{R}^{N} \times(0, \infty),
$$

where $H$ is assumed to be convex and coercive, i.e.,

$$
\inf \{H(x, p)|| x|\leq R,| p \mid \geq r\} \rightarrow+\infty \text { as } r \rightarrow \infty \text { for any } R>0,
$$

and general convergence results for solutions have been established. More precisely, under some appropriate assumptions on the initial value, for the solution $u$ of (1.9), the convergence

$$
u(x, t)-(v(x)-c t) \rightarrow 0 \quad \text { locally uniformly for } x \in \mathbb{R}^{N} \text { as } t \rightarrow \infty
$$

holds, where $(v, c) \in C\left(\mathbb{R}^{N}\right) \times \mathbb{R}$ is a solution of the additive eigenvalue or ergodic problem

$$
H(x, D v(x))=c \quad \text { in } \mathbb{R}^{N} .
$$

Here the additive eigenvalue problem for $H$ is the problem of finding a pair of $v \in C\left(\mathbb{R}^{N}\right)$ and $c \in \mathbb{R}$ such that $v$ is a viscosity solution of (1.11). G. Namah and J.-M. Roquejoffre in [39] were the first to get general results on this convergence under the following additional assumption:

$$
H(x, p) \geq H(x, 0) \text { for all }(x, p) \in \mathcal{M} \times \mathbb{R}^{N} \text { and } \max _{\mathcal{M}} H(x, 0)=0,
$$

where $\mathcal{M}$ is a smooth compact $N$-dimensional manifold without boundary. Independently, A. Fathi [17] proves the same type of convergence result by using the dynamical approach and the weak KAM theory. Contrary to [39, the results of [17] use strict convexity assumptions on $H(x, \cdot)$, i.e., $D_{p p} H(x, p) \geq \alpha I$ for all $(x, p) \in \mathcal{M} \times \mathbb{R}^{N}$ and $\alpha>0$ (and also far more regularity) but do not need (1.12). Afterwards J.-M. Roquejoffre [41] and A. Davini and A. Siconolfi [13] refined the approach of A. Fathi and studied the asymptotic problem for (1.9) on $\mathcal{M}$ or the $N$-dimensional torus. By another approach based on the theory of partial differential equations and viscosity solutions, this type of result has been obtained by G. Barles and P. E. Souganidis in [7. Moreover, we also refer to the literatures [6, 30, 24, 25, 26] for the asymptotic problems without the periodic assumptions and the periodic boundary condition and the literatures [41, 36, 37, 38, 32, 4, 3] for the asymptotic problems which treat HJ equations under various boundary conditions 
including three types of boundary conditions: state constraint boundary condition, Dirichlet boundary condition and Neumann boundary condition. We remark that results in [7, 6, 4, 3] apply to the nonconvex Hamiltonian. It is worth mentioning that as far as the authors know, only the type of convergence (1.10) of solutions of (1.9) has been investigated for HJ equations (1.9).

In our problem, as we explain above, we cannot expect the same type of convergence (1.10). It is due to the noncoercivity of the Hamiltonian $H(x, p)$ given by (1.3). Also, we notice that it is not even convex with respect to the $p$-variable.

1.2. Singular Neumann problems. J.-M. Lasry and P.-L. Lions in 34 provided viscosity formulation of singular boundary conditions for the viscous HJ equation $-\Delta u+|D u|^{p}+\lambda u=f$ in $\Omega$, where $p>1, \lambda>0$ and $f$ is a given smooth function in $\Omega$ and $\Delta u:=\sum_{i=1}^{N} \partial^{2} u / \partial^{2} x_{i}$. Particularly, they proved that if $p>2$, and $f$ satisfies suitable assumptions, there exists a unique solution of the above equation which is continuous on $\bar{\Omega}$ and satisfies the singular Neumann boundary condition (1.6). However, while there are many works on blow-up problems (or singular Dirichlet boundary problems) for (degenerate) elliptic equations, there seem to be very few works which study singular Neumann problems. As far as the authors know, 34 is the only study which investigates the existence and uniqueness of solutions of the singular Neumann problem. Moreover, there are few works for first-order HJ equations. Therefore, the existence, stability and comparison properties for $(\mathrm{N})$ become issues.

We can establish the existence and stability for $(\mathrm{N})$ relatively easily, and the main difficulty lies in the proof of the comparison principle for $(\mathrm{N})$. Here we note that we need to take account of the Aubry-Mather set $\mathcal{A}$ (see [16, 18, 33]) for the uniqueness of viscosity solutions, and therefore the comparison principle for $(\mathrm{N})$ means that if $u \leq v$ on $\mathcal{A}$, then $u \leq v$ on $\bar{\Omega}$, where $u \in C(\bar{\Omega})$ and $v \in \operatorname{LSC}(\bar{\Omega})$ are a subsolution and a supersolution of $(\mathrm{N})$, respectively. It is worth noticing that from the viewpoint of weak KAM theory, the Hamiltonian $F$ has a simple dynamical structure, since the Aubry-Mather set $\mathcal{A}$ for $F-h$ is composed of only the equilibrium point, i.e.,

$$
\mathcal{A}=\left\{x \in \Omega \mid \min _{p \in \mathbb{R}^{N}} F(x, p)-h(x)=0\right\}=\{x \in \Omega \mid h(x)=0\} .
$$

In this paper we give a proof of the comparison principle for $(\mathrm{N})$ in a restrictive case with $F(x, p)=\|p\|$, where $\|\cdot\|$ is a norm in $\mathbb{R}^{N}$. Of course this is restrictive compared with the setting where the existence and the stability of solutions is established. However, this is enough to derive the large-time asymptotics. We postpone this to discuss a more general case in our future work (see Remark 4.4).

1.3. Physical background. We briefly explain a source of our problem coming from the theory of crystal growth. In [44 the morphological stability of a growing faceted crystal is discussed by using a model of a Hamilton-Jacobi equation. The molecularly smooth surfaces of crystals cannot grow without the surface kinetics process, such as the lateral motion of steps and the generation of steps. Such a growth mechanism is usually explained by motion of steps of a macroscopically flat proposed by W. K. Burton, N. Cabrera and F. C. Frank 9]. Its continuum limit gives a macroscopic model described by a Hamilton-Jacobi equation [11. (This type of derivation is rigorously justified by W. E and N. K. Yip [14, and studies of 
the relation between models via step-motion and via macroscopic partial differential equations are still an active research field in various settings; see e.g. 23.)

In a macroscopic model the dimensionless growth speed $V$ in the direction normal to a crystal surface $\Gamma_{t}$ is generally expressed using the surface supersaturation $\sigma(\geq 0)$ and the dimensionless kinetic coefficient $M(p)$ depending on the modulus $p$ of a local slope (gradient) of the crystal surface as in [9] and [12] of the form

$$
\begin{aligned}
& V=M(p) \sigma \\
& M(p)=\frac{p}{p_{s}} \tanh \left(\frac{p_{s}}{p}\right), \\
& p_{s}=\frac{d}{2 x_{s}} .
\end{aligned}
$$

Here $d$ is the step height and $x_{s}$ is the mean surface diffusion distance of a molecule of the surface. The quantity $p_{s}$ is a criterion of local slope which can be regarded as a small parameter and will be denoted by $\varepsilon$. When a surface $\Gamma_{t}$ is given as the graph of a height function $z=z^{\varepsilon}(x, y, t)$, then the equation $V=M(p) \sigma$ is of the form

$$
z_{t}-\sigma(x, y) M_{1}\left(\frac{|D z|}{\varepsilon}\right) \sqrt{1+|D z|^{2}}=0
$$

where $M_{1}(p)=p \tanh (1 / p)$. If one is interested in the behavior of nearly a flat surface, it is natural to assume that $\sigma$ is independent of $z$, so we have assumed that $\sigma$ is independent of $z$ in (1.13).

Unfortunately, if initially $z=0$, then the solution of (1.13) is identically zero and does not grow at all. We need a step source so that the crystal surface $\Gamma_{t}$ grows. It is considered that the kinetic coefficient $M$ is valid only outside the region where there are no step sources.

There are a few ways to include this effect. One way is to modify $M_{1}$ by a continuous, strictly increasing function $m$ on $[0, \infty)$ which agrees with $M_{1}$ except near zero and $m(0)=m_{0}>0$. Another way is to give a growth speed at a particular point of the domain. In [20, the authors added to the equation a step source (driving force) term with jumping discontinuity distributed at one or more points and established the wellposedness by introducing a new notion of viscosity solutions. Moreover, Hamamuki (22]) studied the large-time behavior. In 44] we took the second point of view by putting the growth speed at the step source at the boundary. More precisely, in [4] a one-dimensional Dirichlet problem

$$
z_{t}-\sigma(x) M_{1}\left(\frac{z_{x}}{\varepsilon}\right) \sqrt{1+z_{x}^{2}}=0, \quad x>0, \quad t>0,
$$

with the boundary condition

$$
z(0, t)=c t, \quad t>0,
$$

is considered, where $c>0$ is a fixed constant smaller than $\sigma(0)$.

To explain instability of a facet it is reasonable to consider microscopic time approximation as $\varepsilon \rightarrow 0$ as studied in [44. If one introduces a new dependent variable

$$
\tilde{z}^{\varepsilon}(x, \tau)=z^{\varepsilon}(x, \varepsilon \tau) / \varepsilon
$$


(called a macroscopically flat) and a new independent variable $\tau=t / \varepsilon$ (called a microscopic time), then $\tilde{z}^{\varepsilon}$ converges to a solution of

$$
\left\{\begin{array}{l}
\tilde{z}_{\tau}-\sigma(x) M_{1}\left(\tilde{z}_{x}\right)=0, \quad x>0, \quad \tau>0 \\
\tilde{z}(0, \tau)=c \tau, \quad \tau>0,
\end{array}\right.
$$

as is expected. This is rigorously proved in [44] at least when initially $z=0$ and $\sigma$ is Lipschitz. Note that the Hamiltonian is now noncoercive. When $\sigma$ is a nonincreasing function, it is shown in 44

$$
\tilde{z}(x, \tau) \sim c \tau+b(x) \text { in }\left(0, x_{c}\right) \quad \text { as } \quad \tau \rightarrow \infty
$$

with some computable function $b$ by a method of characteristic and that outside $\left(0, x_{c}\right)$ such a behavior is not expected. The region $\left(0, x_{c}\right)$ is called a stable region in [44, which corresponds to an effective domain in the present paper. Physically speaking, a stable region is a part where a crystal surface stays macroscopically flat. So if a stable region covers all of the crystal surface, the facet is considered to be stable (see the last part of Section 4.5). Otherwise, the facet breaks.

In 21] a more detailed analysis for general initial data as well as for a more general noncoercive Hamiltonian is discussed for the Cauchy-Dirichlet problem in a half line. We note that the notion of a solution in [21] is given in Definition 2 which is shown to be equivalent to our definition (Definition 1) of solutions of the singular Neumann problem (S) as shown in the Appendix 5.2.

In this paper the effect of a step source is included in the Hamiltonian not given as a boundary condition. Namely, we consider the Cauchy problem for

$$
z_{t}-\sigma(x, y) m\left(\frac{|D z|}{\varepsilon}\right) \sqrt{1+|D z|^{2}}=0,
$$

where $m:[0, \infty) \rightarrow(0, \infty)$ is continuous, strictly increasing and equals $M_{1}$ except near the origin and $m(0)=m_{0}>0$. After taking a microscopic time approximation (see Appendix 5.1), we obtain

$$
\tilde{z}_{\tau}-\sigma(x, y) m(|D \tilde{z}|)=0 .
$$

If one introduces $u=-\tilde{z}$ with $t=\tau$, we end up with

$$
u_{t}+\sigma(x, y) m(|D u|)=0 .
$$

In this case we see that the effective domain $\Omega_{e}$ is given by $\Omega_{e}:=\{(x, y) \mid$ $\left.\sigma(x, y)>\bar{\sigma} m_{0}\right\}$, where we denote the maximum of $\sigma$ by $\bar{\sigma}$. Our main result in particular implies that under the convexity and boundedness assumption on $\Omega_{e}$ and the nondegeneracy of $D \sigma$ on $\partial \Omega_{e}$, i.e., $D \sigma \neq 0$ on $\partial \Omega_{e}$, we have

$$
u(x, y, t) \sim-c t+v_{\infty}(x, y) \text { in } \Omega_{e} \text { as } t \rightarrow \infty,
$$

where $v_{\infty}$ is a solution of the corresponding singular Neumann problem

$$
\begin{cases}|D u|=h(x, y):=m^{-1}\left(\frac{\bar{\sigma} m_{0}}{\sigma(x, y)}\right) & \text { in } \Omega_{e}, \\ \frac{\partial u}{\partial n}=+\infty & \text { on } \partial \Omega_{e} \\ \sup _{(x, y) \in \Omega_{e}}|u(x, y)|<+\infty . & \end{cases}
$$

Outside the closure of $\Omega_{e}$ the function $u$ tends to $+\infty$ as $t \rightarrow \infty$. Thus, physically $\Omega_{e}$ is a stable region where a crystal surface stays macroscopically flat. It is worth mentioning that the Aubry-Mather set of the corresponding stationary problem is 
considered as the set of step sources which consists of all global maximum points of $\sigma$. More precise descriptions are given in Section 4.5.

This paper is organized as follows. In the next section, we consider the largetime behavior of solutions of (1.4) by the method of characteristics. In Section 3, we consider singular Neumann problems (N) and establish existence, stability and comparison results. Section 4 is devoted to giving the large-time asymptotic profile of viscosity solutions of $(\mathrm{C})$. In the Appendix, we give an explanation for the derivation of (1.1), another characterization of solutions of $(\mathrm{N})$ and proofs for some propositions used in Section 2.

Notation. Let $\mathbb{R}^{k}$ denote the $k$-dimensional Euclidean space for some $k \in \mathbb{N}$. We write $B(x, r)=\left\{y \in \mathbb{R}^{k}|| x-y \mid<r\right\}$ and $[x, y]:=\{\lambda x+(1-\lambda) y \mid \lambda \in[0,1]\}$ for $x, y \in \mathbb{R}^{k}$ and $r>0$. We denote by $|\cdot|$ the usual Euclidean norm. For $A, B \subset$ $\mathbb{R}^{k}$, we denote by $C(A, B)$ and $\mathrm{UC}(A, B)$ the space of continuous and uniformly continuous functions on $A$ with values in $B$, respectively. We denote by $\operatorname{LSC}(A)$, $\operatorname{USC}(A), \operatorname{Lip}(A)$ and $C^{k}(A)$ the space of real-valued lower semicontinuous, upper semicontinuous, Lipschitz continuous, and $k$-th continuous differentiable functions on $A$ and we write $C^{\infty}(A)=\bigcap_{k \in \mathbb{N}} C^{k}(A)$. For $a, b \in \mathbb{R}$, we write $a \wedge b=\min \{a, b\}$ and $a \vee b=\max \{a, b\}$. We call a function $m:[0, \infty) \rightarrow[0, \infty)$ a modulus if it is continuous and nondecreasing on $[0, \infty)$ and if $m(0)=0$.

\section{A BASIC EXAMPLE IN ONE-DIMENSION}

In this section we investigate the one-dimensional Cauchy problem (1.4) and we see that the large-time behavior of the viscosity solution of $(\mathrm{C})$ is completely different from the case where the Hamiltonian is assumed to be coercive, which is explained in the Introduction.

In order to solve this problem by the method of characteristics, we first consider the initial boundary value problem

$$
\begin{cases}v_{t}+\frac{2}{\pi} \arctan \left(v_{x}^{2}\right)=x & \text { in }(0, \infty) \times(0, \infty), \\ v(0, t)=0 & \text { for all } t \in[0, \infty), \\ v(x, 0)=0 & \text { for all } x \in[0, \infty) .\end{cases}
$$

We have the Hamiltonian system for this problem,

$$
\begin{cases}\frac{d x(t)}{d t}=\frac{4 p(t)}{\pi\left(1+p(t)^{4}\right)} & \text { for } t \in(0, \infty), \\ \frac{d p(t)}{d t}=1 & \text { for } t \in(0, \infty), \\ x(0)=x_{0} \in \mathbb{R}, p(0)=0, & \end{cases}
$$

and its solution could be explicitly calculated as

$$
x(t)=x_{0}+\frac{2}{\pi} \arctan \left(t^{2}\right) \text { and } p(t)=t .
$$

Setting $z(t)=v(x(t), t)$, then it is satisfied that

$$
\frac{d z(t)}{d t}=\frac{4 t^{2}}{\pi\left(1+t^{4}\right)}+x(t)-\frac{2}{\pi} \arctan \left(t^{2}\right)=\frac{4 t^{2}}{\pi\left(1+t^{4}\right)}+x_{0} .
$$

Notice that the first term on the far right hand side of (2.2) is integrable and $x_{0}$ should be positive when we have $x(t)>1$ for some $t>0$. Moreover, an observation 
is that given any $x$ greater than 1 and $t$ sufficiently large, if we can solve the characteristics inversely from $x(t)=x$ to find $x_{0}$, the curve that starts from $x_{0}$ will cross the line $x=1$ and stay in the region $x>1$ for a very long time until it reaches the point $x$. Putting this observation into (2.2), we find $z(t)=v(x(t), t)$ tends to plus infinity as $t$ goes to infinity if $x>1$.

As a result, we see that the large-time asymptotic profile $v_{\infty}$ of the solution $v$ of (2.1) is

$$
v_{\infty}(x)= \begin{cases}\int_{0}^{x}\left(\tan \left(\frac{\pi}{2} y\right)\right)^{1 / 2} d y & \text { for all } x \in[0,1] \\ +\infty & \text { for all } x \in(1, \infty) .\end{cases}
$$

Finally, we note that the function $u$ defined by

$$
u(x, t)= \begin{cases}v(x, t) & \text { for all }(x, t) \in[0, \infty) \times[0, \infty), \\ v(-x, t) & \text { for all }(x, t) \in(-\infty, 0] \times[0, \infty)\end{cases}
$$

is a unique viscosity solution of (1.4). We only need to check that $u$ satisfies the equation (1.4) at $x=0$ in the viscosity sense. Indeed, the subdifferential of $u$ at $x=0$ and any $t>0$ is an empty set and the superdifferential of $u$ with respect to the $t$-variable is 0 . Thus any $p \in \mathbb{R}$ satisfies $0+(2 / \pi) \arctan \left(p^{2}\right) \geq 0$, which implies that $u$ is a viscosity supersolution at $x=0$.

Therefore, we see that the large-time asymptotic profile $u_{\infty}$ of the solution $u$ of (1.4) is

$$
u_{\infty}(x)= \begin{cases}\int_{0}^{|x|}\left(\tan \left(\frac{\pi}{2} y\right)\right)^{1 / 2} d y & \text { for all } x \in[-1,1] \\ +\infty & \text { for all } x \in(-\infty,-1) \cup(1, \infty)\end{cases}
$$

\section{Singular Neumann PRoblems}

In this section we consider singular Neumann problems $(\mathrm{N})$ and we present the definition, existence, stability and comparison results of solutions of $(\mathrm{N})$.

We shall use the following assumptions in this section:

(A1) $F \in C\left(\bar{\Omega} \times \mathbb{R}^{N}\right)$ and $h \in C(\Omega)$ with $h \geq 0$ in $\Omega$.

(A2) $F$ is coercive with respect to the $p$-variable uniformly for $x \in \bar{\Omega}$, i.e.,

$$
\inf \{F(x, p)|x \in \bar{\Omega},| p \mid \geq r\} \rightarrow+\infty \text { as } r \rightarrow \infty .
$$

(A3) For any $x \in \bar{\Omega}, p \mapsto F(x, p)$ are convex functions.

(A4) $F(x, p) \geq F(x, 0)=0$ for any $(x, p) \in \bar{\Omega} \times \mathbb{R}^{N}$.

(A5) The set $\mathcal{A}_{h} \neq \emptyset$, where $\mathcal{A}_{h}:=\{x \in \bar{\Omega} \mid h(x)=0\}$.

(A6) $h(x) \rightarrow \infty$ as $\operatorname{dist}(x, \partial \Omega) \rightarrow 0$.

(A7) There exists a modulus $\omega$ such that

$$
\int_{0}^{1} h(s x+(1-s) y)|x-y| d s \leq \omega(|x-y|)
$$

for all $x, y \in \Omega$ such that $[x, y] \subset \Omega$.

(A8) $\Omega$ is a bounded domain and $\partial \Omega$ is locally represented as the graph of a continuous function, i.e., for each $z \in \partial \Omega$ there exist $r>0$ and a function $b \in C\left(\mathbb{R}^{n-1}\right)$ such that - upon relabelling and re-orienting the coordinates axes if necessary - we have

$$
\Omega \cap B(z, r)=\left\{\left(x^{\prime}, x_{n}\right) \in \mathbb{R}^{n-1} \times \mathbb{R} \mid x \in B(z, r), x_{n}>b\left(x^{\prime}\right)\right\} .
$$


Remark 3.1. Assumption (A7) is a kind of growth condition on $h$. In the case where $N=1$, if $h \in C((a, b)) \cap L^{1}(a, b)$ with $a<b$, then (A7) holds. Indeed, setting

$$
\omega(r):=\sup _{x \in(a, b)} \int_{x}^{x+r} \bar{h}(s) d s,
$$

where $\bar{h}: \mathbb{R} \rightarrow \mathbb{R}$ is the function defined by $\bar{h}(x)=h(x)$ for all $s \in(a, b)$ and $\bar{h}(x)=0$ for all $s \in \mathbb{R} \backslash(a, b)$, we have

$$
\int_{0}^{1}|h(s x+(1-s) y)||x-y| d s \leq\left|\int_{y}^{x}\right| h(r)|d r| \leq \omega(|x-y|)
$$

for any $x, y \in \mathbb{R}$ with $x \neq y$. In the case where $N \geq 2$, the integrability does not imply (A7) in general.

3.1. Definition, existence and stability. We use the following definition of solutions of (N) introduced in [34, Section V.1].

Definition 1 (Definition of solutions of $(\mathrm{N})$ ). Let $u$ be a function on $\bar{\Omega}$ with values in $\mathbb{R}$. We call $u$ a subsolution of $(\mathrm{N})$ if $u \in \operatorname{USC}(\bar{\Omega})$ is a viscosity subsolution of (1.5). We call $u$ a supersolution of $(\mathrm{N})$ if $u \in \operatorname{LSC}(\bar{\Omega})$ is a viscosity supersolution of (1.5) and satisfies (1.8). We call $u$ a solution of $(\mathrm{N})$ if $u \in C(\bar{\Omega})$ is a subsolution and a supersolution of $(\mathrm{N})$.

Remark 3.2. It is worth mentioning that we do not require any condition for subsolutions on the boundary, which is really like the state constraint boundary condition introduced in [42].

Theorem 3.1 (Existence of solutions of (1.5) satisfying (1.8)). Assume that (A1)(A6) hold. There exists a viscosity solution $u \in C(\Omega)$ of (1.5) which satisfies (1.8).

Remark 3.3. We remark that solutions of (1.5) can blow up at some boundary point, if we do not assume (A7). Indeed, when we consider

$$
\left|u_{x}\right|=\frac{1}{x^{2}} \text { in }(0,1)
$$

then it is clear that the right hand side does not satisfy (A7) and any solution $u$ blows up at $x=0$, i.e., $u(0)=+\infty$.

Proof of Theorem 3.1. Take any function $w \in C^{1}(\Omega)$ such that

$$
\begin{aligned}
& w(x) \geq 0 \quad \text { for all } x \in \Omega, \\
& w(x)=0 \text { for all } x \in \mathcal{A}_{h}, \\
& w(x) \rightarrow+\infty \quad \text { as } \operatorname{dist}(x, \partial \Omega) \rightarrow 0 .
\end{aligned}
$$

Set

$$
w_{\varepsilon}(x):=\varepsilon w(x) \text { and } h_{\varepsilon}(x):=F\left(x, D w_{\varepsilon}(x)\right) .
$$

Since $D w_{\varepsilon}(x)=\varepsilon D w(x) \rightarrow 0$ locally uniformly in $x \in \Omega$ as $\varepsilon \rightarrow 0$ and $F(x, 0)=0$ for all $x \in \bar{\Omega}$, we have $h_{\varepsilon} \rightarrow 0$ locally uniformly in $x \in \Omega$. It is clear that $h_{\varepsilon} \in C(\Omega)$ and $h_{\varepsilon}(x) \geq 0$ for all $x \in \Omega$. Note that

$$
F\left(x, D w_{\varepsilon}(x)\right)=h_{\varepsilon}(x) \leq h(x)+h_{\varepsilon}(x) \text { in } \Omega .
$$


We define the functions $u_{\varepsilon}^{\delta} \in C\left(\bar{\Omega}_{\delta}\right)$ by

$$
u_{\varepsilon}^{\delta}(x):=\min \left\{d_{\varepsilon}(x, y)+w_{\varepsilon}(y) \mid y \in \partial \Omega_{\delta} \cup \mathcal{A}_{h}\right\}
$$

for small $\delta>0$, where

$$
\begin{aligned}
& d_{\varepsilon}(x, y):=\sup \left\{v(x)-v(y) \mid F(x, D v) \leq h(x)+h_{\varepsilon}(x) \text { in } \Omega\right\}, \\
& \Omega_{\delta}:=\{x \in \Omega \mid \operatorname{dist}(x, \partial \Omega)>\delta\} .
\end{aligned}
$$

Note that $h_{\varepsilon}(x)=0$ for all $x \in \mathcal{A}_{h}$ and therefore we have $\mathcal{A}_{h}=\{x \in \Omega \mid h(x)+$ $\left.h_{\varepsilon}(x)=0\right\}$. Since the functions $w_{\varepsilon}$ satisfy the compatibility condition

$$
w_{\varepsilon}(x)-w_{\varepsilon}(y) \leq d_{\varepsilon}(x, y) \text { for all } x, y \in \partial \Omega_{\delta} \cup \mathcal{A}_{h},
$$

in view of [33, Theorem 3.3] we see that $u_{\varepsilon}^{\delta}$ is a unique viscosity solution of

$$
F(x, D u)=h(x)+h_{\varepsilon}(x) \quad \text { in } \Omega_{\delta}
$$

which satisfies

$$
u_{\varepsilon}^{\delta}(x)=w_{\varepsilon}(x) \quad \text { for all } x \in \partial \Omega_{\delta} \cup \mathcal{A}_{h} .
$$

We extend $u_{\varepsilon}^{\delta}$ to a continuous function in $\Omega$ and denote it by $u_{\varepsilon}^{\delta}$ again.

The comparison result for (3.2) and (3.3) (see [33, Theorem 7.3]) implies that

$$
(0 \leq) w_{\varepsilon} \leq u_{\varepsilon}^{\delta} \text { on } \bar{\Omega}_{\delta} \text {. }
$$

Fix $\delta_{0}>0$ so small that $\mathcal{A}_{h} \subset \Omega_{\delta_{0}}$. For any $\delta<\delta_{0}$, we have

$$
F\left(x, D u_{\varepsilon}^{\delta}\right)=h(x)+h_{\varepsilon}(x) \leq \max _{\bar{\Omega}_{\delta_{0}}}\left(h+h_{\varepsilon}\right)(x) \quad \text { in } \Omega_{\delta_{0}}
$$

in the viscosity sense. In view of the coercivity of $F$, we have

$$
\left|D u_{\varepsilon}^{\delta}(x)\right| \leq C_{\delta_{0}} \quad \text { in } \Omega_{\delta_{0}}
$$

for some $C_{\delta_{0}}$ and any $\delta \in\left(0, \delta_{0}\right)$. Therefore, we obtain the equi-continuity of $\left\{u_{\varepsilon}^{\delta}\right\}_{0<\delta<\delta_{0}}$ in $\Omega_{\delta_{0}}$. Since $u_{\varepsilon}^{\delta}=w_{\varepsilon}=0$ on $\mathcal{A}_{h},\left\{u_{\varepsilon}^{\delta}\right\}_{0<\delta<\delta_{0}}$ is uniformly bounded in $\Omega_{\delta_{0}}$. Noting that $u_{\varepsilon}^{\delta_{1}}=u_{\varepsilon}^{\delta_{2}}=w_{\varepsilon}$ on $\mathcal{A}_{h}, u_{\varepsilon}^{\delta_{2}}=w_{\varepsilon}$ on $\partial \Omega_{\delta_{2}}$, and $u_{\varepsilon}^{\delta_{1}} \geq w_{\varepsilon}$, we get $u_{\varepsilon}^{\delta_{2}} \leq u_{\varepsilon}^{\delta_{1}}$ on $\bar{\Omega}_{\delta_{2}}$ for any small $\delta_{1}, \delta_{2}$ with $\delta_{1}<\delta_{2}$ by the comparison principle [33, Theorem 7.3] again. Therefore, $u_{\varepsilon}^{\delta}$ converges to some $u_{\varepsilon} \in C(\Omega)$ locally uniformly in $\Omega$ as $\delta \rightarrow 0$, which is a solution of $F(x, D u)=h(x)+h_{\varepsilon}(x)$ in $\Omega$. Moreover, $u_{\varepsilon}$ satisfies

$$
u_{\varepsilon}(x) \rightarrow+\infty \quad \text { as } \operatorname{dist}(x, \partial \Omega) \rightarrow 0,
$$

since $u_{\varepsilon} \geq w_{\varepsilon}$ in $\Omega$.

Let $K$ be a compact set in $\Omega$. Then we have

$$
\left|D u_{\varepsilon}\right| \leq C_{K} \text { in int } K \text { in the viscosity sense and }\left|u_{\varepsilon}\right| \leq C_{K} \text { on } K
$$

for some $C_{K}>0$ which depends only on $K$, which implies that $\left\{u_{\varepsilon}\right\}_{\varepsilon>0}$ is relatively compact in $C(\Omega)$. Therefore, there exists $\left\{u_{\varepsilon_{j}}\right\}_{j \in \mathbb{N}} \subset\left\{u_{\varepsilon}\right\}_{\varepsilon>0}$ such that $u_{\varepsilon_{j}} \rightarrow u$ locally uniformly in $\Omega$ as $j \rightarrow \infty$ for some $u \in C(\Omega)$, which satisfies $F(x, D u)=h(x)$ in $\Omega$ in the viscosity sense. We define the function $\underline{u} \in \operatorname{LSC}(\bar{\Omega})$ by

$$
\underline{u}(x):= \begin{cases}u(x) & \text { for all } x \in \Omega, \\ \liminf _{y \rightarrow x, y \in \Omega} u(y) & \text { for all } y \in \partial \Omega .\end{cases}
$$

We finally prove that $\underline{u}$ satisfies (1.8). Let $x_{0} \in \partial \Omega$ and then we can distinguish two cases: (i) $\underline{u}\left(x_{0}\right)=+\infty$; (ii) $-\infty<\underline{u}\left(x_{0}\right)<+\infty$. In case (i), it is easy to check that $\underline{u}$ satisfies (1.8). 
In case (ii), let $\phi \in C^{1}(\bar{\Omega})$ and $x_{0}$ be a strict minimum over $\bar{\Omega}$ of $\underline{u}-\phi$. Since $u_{\varepsilon_{j}}(x) \rightarrow \infty$ as $\operatorname{dist}(x, \partial \Omega) \rightarrow 0, u_{\varepsilon_{j}}-\phi$ has a minimum over $\Omega$ at some point $x_{\varepsilon_{j}} \in \Omega$ and $x_{\varepsilon_{j}} \rightarrow x_{0}$ as $j \rightarrow \infty$. We have

$$
F\left(x_{\varepsilon_{j}}, D \phi\left(x_{\varepsilon_{j}}\right)\right) \geq h\left(x_{\varepsilon_{j}}\right) .
$$

The above inequality yields a contradiction for a suitable small $\varepsilon>0$, since $h(x) \rightarrow$ $\infty$ as dist $(x, \partial \Omega) \rightarrow 0$ and $|D \phi(x)|<+\infty$ for all $x \in \bar{\Omega}$.

The above idea of using the function $w$ which blows up at any boundary point is used for the proof of approximations of solutions of the stationary state constraint problem in [10, Theorem VII.3], and we also refer to [34] in different problems.

Theorem 3.2 (Existence of solutions of $(\mathrm{N}))$. Assume that (A1)-(A8) hold. There exists a solution $u \in C(\bar{\Omega})$ of $(\mathrm{N})$.

Lemma 3.3 (Uniform continuity of subsolutions). Assume that (A1)-(A3), (A7) and (A8) hold. Let $u \in \operatorname{USC}(\bar{\Omega})$ be a subsolution of (1.5). Then $u$ is uniformly continuous in $\Omega$.

We give the proof of the above lemma in Appendix 5.3.

Proof of Theorem 3.2. Let $u$ be the function given in the proof of Theorem 3.1. By Lemma 3.3, $u$ is uniformly continuous in $\Omega$. Therefore, $u$ can be extended uniquely to a function on $\bar{\Omega}$ by continuity and it is a solution of $(\mathrm{N})$ as a continuous function on $\bar{\Omega}$.

We give a stability result under infimum operation. It is worth mentioning that the stability property, without boundary condition, is the main technical observation in the theory of lower semicontinuous viscosity solutions due to E. N. Barron and R. Jensen [8]. We remark that H. Ishii gives this stability result (see [31, Theorem 2.1]) under the oblique Neumann condition.

Theorem 3.4 (Stability under the inf-operation). Assume that (A1), (A3) and (A6) hold. Let $\mathcal{S} \subset C(\bar{\Omega})$ be a nonempty subset of solutions of $(\mathrm{N})$. Set

$$
u(x):=\inf \{v(x) \mid v \in \mathcal{S}\} .
$$

If $u$ is continuous on $\bar{\Omega}$, then $u$ is a solution of $(\mathrm{N})$.

Proof. By the standard stability property of viscosity solutions, we see that $u$ is a supersolution of (1.5). Moreover, in view of the convexity of Hamiltonian, we see that $u$ is a subsolution of (1.5), and therefore we only need to prove that $u$ satisfies the boundary condition. We argue by contradiction. Suppose that there would exist $\phi \in C^{1}(\bar{\Omega})$ and $z \in \partial \Omega$ such that $u-\phi$ takes a strict minimum at $z$.

By the definition of $u$, for any $k \in \mathbb{N}$, there exists $v_{k} \in \mathcal{S}$ such that $u(z)>v_{k}(z)-$ $1 / k$. We choose a sequence $\left\{y_{k}\right\}_{k \in \mathbb{N}} \subset \bar{\Omega}$ satisfying $\left(v_{k}-\phi\right)\left(y_{k}\right)=\min _{\bar{\Omega}}\left(v_{k}-\phi\right)$. Since $v_{k}$ is a solution of $(\mathrm{N})$, we have $\left\{y_{k}\right\}_{k \in \mathbb{N}} \subset \Omega$. Noting that

$$
(u-\phi)(z)+\frac{1}{k}>\left(v_{k}-\phi\right)(z) \geq\left(v_{k}-\phi\right)\left(y_{k}\right) \geq(u-\phi)\left(y_{k}\right) \geq(u-\phi)(z),
$$


we have $(u-\phi)\left(y_{k}\right) \rightarrow(u-\phi)(z)$ as $k \rightarrow \infty$. Since $u-\phi$ takes a strict minimum at $z$, we obtain $y_{k} \rightarrow z$ as $k \rightarrow \infty$. The definition of viscosity solutions implies that

$$
F\left(y_{k}, D \phi\left(y_{k}\right)\right) \geq h\left(y_{k}\right) \text {. }
$$

We have $h\left(y_{k}\right) \rightarrow+\infty$ as $k \rightarrow \infty$, contradicting the boundedness of $F\left(y_{k}, D \phi\left(y_{k}\right)\right)$.

We define the function $d$ on $\Omega \times \Omega$ by

$$
d(x, y):=\sup \{v(x)-v(y) \mid F(x, D v) \leq h(x) \text { in } \Omega \text { in the viscosity sense }\} .
$$

To see that $d$ is well defined, we set $\mathcal{S}^{-}:=\{v-v(y) \mid v$ is a subsolution (1.5) $\}$. Note that, due to (A4), $\mathcal{S}^{-}$is nonempty. By coercivity (A2) $\mathcal{S}^{-}$is a family of equi-Lipschitz continuous functions locally in $\Omega$. Note also that $\phi(y)=0$ for all $\phi \in \mathcal{S}^{-}$and that $\Omega$ is connected. Therefore, thanks to the Ascoli-Arzelà theorem, $\mathcal{S}^{-}$is precompact in the Fréchet space $C(\Omega)$. Thus the function $d$ is a continuous function on $\Omega \times \Omega$ and satisfies $d(x, x)=0$ for all $x \in \Omega$. Furthermore, by definition, we have $u(x)-u(y) \leq d(x, y)$ for all subsolutions $u$ of (1.5) and $x, y \in \Omega$.

Now, we fix any $y \in \Omega$ and set $u(x)=d(x, y)$ for $x \in \Omega$. We see that $u$ is locally Lipschitz continuous on $\Omega$ in view of (A2) and $u$ is a subsolution (1.5). We argue as in the proof of Perron's method for viscosity solutions (see [28, 1, 19]), to find that $u$ is a solution of (1.5) except at $\{y\}$. Next, we note by the definition of $d$ that $v(x)-v(y)=v(x)-v(z)+v(z)-v(y) \leq d(x, z)+d(x, z)$ for all subsolutions $v$ of (1.5) and $x, y, z \in \Omega$, to conclude that $d(x, y) \leq d(x, z)+d(z, y)$ for all $x, y, z \in \Omega$. In particular, we see that $d$ is locally Lipschitz continuous on $\Omega \times \Omega$. The following proposition summarizes these observations.

Proposition 3.5. Assume that (A1), (A2) and (A4) hold. Then the following statements hold. (i) $d(x, x)=0$ for all $x \in \Omega$ and $d$ is locally Lipschitz continuous on $\Omega \times \Omega$. (ii) For all $y \in \Omega, d(\cdot, y)$ is a subsolution of (1.5). (iii) For all $y \in \Omega$, $d(\cdot, y)$ is a solution of (1.5) except at $\{y\}$. (iv) $d(x, y) \leq d(x, z)+d(z, y)$ for all $x, y, z \in \Omega$.

It is well known that we have a variational formula for the function $d$, i.e.,

$$
d(x, y)=\inf \left\{\int_{0}^{t} L(\gamma(s), \dot{\gamma}(s))+h(\gamma(s)) d s \mid t>0, \gamma \in \mathcal{C}(x, t ; y, 0)\right\}
$$

for any $x, y \in \Omega$, where $L$ is the Lagrangian of $F$, i.e., $L(x, \xi)=\sup _{p \in \mathbb{R}^{N}}\{p \cdot \xi-$ $F(x, p)\}$,

$$
\mathcal{C}(x, t ; y, 0):=\{\gamma \in \mathrm{AC}([0, t] ; \Omega) \mid \gamma(t)=x, \gamma(0)=y\}
$$

and we denote the set of absolutely continuous functions on $[0, t]$ with values in $\Omega$ by $\mathrm{AC}([0, t] ; \Omega)$. For a proof, we refer the reader to [35, 1, 30].

Proposition 3.6. Assume that (A1)-(A3), (A7) and (A8) hold. The function d is uniformly continuous in $\Omega \times \Omega$.

We give the proof of the above proposition in Appendix 5.3 and the function $d$ can be extended uniquely to the function on $\bar{\Omega} \times \bar{\Omega}$ by continuity. We denote it by $d$ again.

Proposition 3.7. Assume that (A1)-(A8) hold. Then the following statements hold. (i) For any $y \in \bar{\Omega}, d(\cdot, y)$ is a supersolution of $(\mathrm{N})$ except at $\{y\}$. (ii) For any $y \in \mathcal{A}_{h}, d(\cdot, y)$ is a solution of $(\mathrm{N})$. 
Proof. We first prove (i). Fix $y \in \bar{\Omega}$ and set $u(x):=d(x, y)$ for all $x \in \bar{\Omega}$. We only need to prove that $d(\cdot, y)$ satisfies the boundary condition defined by Definition 1 except at the point $y$. We argue by contradiction. Suppose that there would exist a test function $\phi \in C^{1}(\bar{\Omega})$ such that $u-\phi$ takes a strict minimum at some $z \in \partial \Omega$ with $z \neq y$. We may assume that $(u-\phi)(z)=0$. Noting that

$$
F(z, D \phi(z))<+\infty \text { and } h(z)=+\infty
$$

we have

$$
\begin{gathered}
F(x, D \phi(x)) \leq h(x) \quad \text { for all } x \in \bar{\Omega} \cap B(z, r), \\
y \notin \bar{\Omega} \cap \bar{B}(z, r)
\end{gathered}
$$

for some $r>0$.

Set $m:=\min _{x \in \bar{\Omega} \cap \partial B(z, r)}(u-\phi)(x)$. Then we see that $m>0$. For any $t>0$ and $\gamma \in \mathcal{C}(z, t ; y, 0)$, there exists $\tau \in(0, t)$ such that

$$
\begin{gathered}
\gamma(\tau) \in \bar{\Omega} \cap \partial B(z, r), \\
\gamma(s) \in \bar{\Omega} \cap B(z, r) \quad \text { for all } s \in[\tau, t] .
\end{gathered}
$$

We calculate that

$$
\begin{aligned}
u(z)= & \phi(\gamma(t))=\phi(\gamma(\tau))+\int_{\tau}^{t} \frac{d \phi(\gamma(s))}{d s} d s \\
= & \phi(\gamma(\tau))+\int_{\tau}^{t} D \phi(\gamma(s)) \cdot \dot{\gamma}(s) d s \\
\leq & u(\gamma(\tau))-m \\
& +\int_{\tau}^{t}(F(\gamma(s), D \phi(\gamma(s)))-h(\gamma(s)))+(L(\gamma(s), \dot{\gamma}(s))+h(\gamma(s))) d s \\
\leq & \int_{0}^{\tau} L(\gamma(s), \dot{\gamma}(s))+h(\gamma(s)) d s+\int_{\tau}^{t} L(\gamma(s), \dot{\gamma}(s))+h(\gamma(s)) d s-m \\
= & \int_{0}^{t} L(\gamma(s), \dot{\gamma}(s))+h(\gamma(s)) d s-m .
\end{aligned}
$$

Therefore, we get $u(z) \leq u(z)-m$, which is a contradiction.

We next prove (ii). Fix $y \in \mathcal{A}_{h}$. We just need to prove that $d$ is a viscosity supersolution at the point $y$. Suppose that there would exist a test function $\phi \in$ $C^{1}(\bar{\Omega})$ such that

$$
\begin{gathered}
d(\cdot, y)-\phi \text { takes a local minimum at } y \text { and } \\
F(y, D \phi(y))<h(y) .
\end{gathered}
$$

It is easy to see that the above inequality has a contradiction, since $F(x, p) \geq 0$ for all $(x, p) \in \bar{\Omega} \times \mathbb{R}^{N}$ and $h(y)=0$.

3.2. Comparison result. We prove that the comparison principle for $(\mathrm{N})$ holds in a special case. In this subsection, we deal with the Hamiltonian defined by

$$
F(x, p)=\|p\|,
$$

where $\|\cdot\|$ is a norm in $\mathbb{R}^{N}$.

We add the following assumption on $h$ and $\Omega$. 
(A9) There exists a constant $\alpha_{0}>0$ such that $\{x \in \Omega \mid h(x)<\alpha\}$ are convex for all $\alpha \geq \alpha_{0}$. (This forces $\Omega$ to be convex.)

Theorem 3.8 (Comparison principle for $(\mathrm{N}))$. Let $F$ be as in (3.5). Assume (A1), (A5), (A6) and (A9) hold. Let $u \in C(\bar{\Omega})$ and $v \in \operatorname{LSC}(\bar{\Omega})$ be a subsolution and a supersolution of $(\mathrm{N})$, respectively. If $u \leq v$ on $\mathcal{A}_{h}$, then $u \leq v$ on $\bar{\Omega}$.

Remark 3.4. If we do not require the continuity up to the boundary of $u$, then it may happen that $u\left(x_{0}\right)>v\left(x_{0}\right)$ at some point $x_{0} \in \partial \Omega$, since there is no requirement for subsolutions on the boundary. Therefore we cannot remove the continuity assumption on $u$ in Theroem 3.8 .

For the proof of the above theorem we need an anisotropic Lipschitz extension lemma (Lemma 3.9) and a regularization lemma (Lemma 3.10).

Lemma 3.9 (Anisotropic Lipschitz extension). Assume that (A1) and (A9) hold. Let $u \in C(\bar{\Omega})$ be a subsolution of $(\mathrm{N})$. Define the functions $u_{n}: \mathbb{R}^{N} \rightarrow \mathbb{R}$ by

$$
u_{n}(x):=\max _{y \in \bar{\Omega}_{n}}\left\{u(y)-n\|x-y\|_{*}\right\},
$$

for any $n \in \mathbb{N}$, where $\Omega_{n}:=\{x \in \Omega \mid h(x)<n\}$ and $\|\cdot\|_{*}$ is the dual norm of $\|\cdot\|$, i.e.,

$$
\|q\|_{*}:=\sup _{p \in \mathbb{R}^{N} \backslash\{0\}} \frac{|p \cdot q|}{\|p\|} .
$$

Let $\alpha_{0}$ be the constant appearing in (A9). Then for all $n \geq \alpha_{0}$ we have

$$
\begin{gathered}
u_{n}(x)=u(x) \quad \text { for all } x \in \bar{\Omega}_{n}, \\
\left|u_{n}(x)-u_{n}(y)\right| \leq n\|x-y\|_{*} \text { for all } x, y \in \mathbb{R}^{N}, \\
\left\|D u_{n}\right\| \leq n \quad \text { in } \mathbb{R}^{N} \text { in the viscosity sense. }
\end{gathered}
$$

Moreover, $u_{n}$ are subsolutions of $(\mathrm{N})$ for all $n \in \mathbb{N}$ with $n \geq \alpha_{0}$ and $u_{n} \rightarrow u$ as $n \rightarrow \infty$ in $C(\bar{\Omega})$.

Remark 3.5. When $\|\cdot\|=|\cdot|$, the functions $u_{n}$ are known as extensions of Lipschitz functions. See Theorem 1 (p. 80) in [15] for instance. We use the continuity of $u$ on $\bar{\Omega}$ in the convergence of $u_{n}$ to $u$ in $C(\bar{\Omega})$.

Proof. By the definition of $\Omega_{n}$ we have

$$
\|D u\| \leq n \quad \text { in } \Omega_{n}
$$

in the viscosity sense. In view of (A9) and Proposition 5.8, we have

$$
|u(x)-u(y)| \leq n\|x-y\|_{*} \text { for all } x, y \in \bar{\Omega}_{n}, n \geq \alpha_{0},
$$

which implies that $u_{n}(x) \leq u(x)$ for all $x \in \bar{\Omega}_{n}$. It is clear that $u_{n}(x) \geq u(x)$ for any $x \in \bar{\Omega}_{n}$. Thus we get $u_{n}=u$ in $\bar{\Omega}_{n}$.

For any $x_{1}, x_{2} \in \mathbb{R}^{N}$, we have

$$
\begin{aligned}
u_{n}\left(x_{1}\right) & =\max _{y \in \bar{\Omega}_{n}}\left\{u(y)-n\left\|x_{1}-y\right\|_{*}\right\} \\
& \geq \max _{y \in \bar{\Omega}_{n}}\left\{u(y)-n\left\|x_{1}-x_{2}\right\|_{*}-n\left\|x_{2}-y\right\|_{*}\right\} \\
& =u_{n}\left(x_{2}\right)-n\left\|x_{1}-x_{2}\right\|_{*} .
\end{aligned}
$$


By symmetry we get

$$
\left|u_{n}\left(x_{1}\right)-u_{n}\left(x_{2}\right)\right| \leq n\left\|x_{1}-x_{2}\right\|_{*} .
$$

By Proposition 5.5 we have

$$
\left\|D u_{n}(x)\right\| \leq n \quad \text { in } \mathbb{R}^{N} \text { in the viscosity sense. }
$$

We next prove that $u_{n}$ are subsolutions of $(\mathrm{N})$ for all $n \geq \alpha_{0}$. For any $x \in \Omega_{n}$, we have $u_{n}(x)=u(x)$. Therefore, we have $\|p\| \leq h(x)$ for any $p \in D^{+} u_{n}(x)=D^{+} u(x)$, since $u$ is a viscosity subsolution in $\Omega$. For any $x \in \Omega \backslash \Omega_{n}$, we have $h(x) \geq n$. Noting that inequality (3.8) implies that $\|p\| \leq n$ for all $p \in D^{+} u_{n}(x)$, we obtain

$$
\|p\|-h(x) \leq n-n=0
$$

for all $x \in \Omega \backslash \Omega_{n}$ and all $p \in D^{+} u_{n}(x)$. Finally, since we have $u_{n}=u$ in $\Omega_{n}$ and $u \in C(\bar{\Omega})$, we see that $u_{n} \rightarrow u$ as $n \rightarrow \infty$ in $C(\bar{\Omega})$.

Lemma 3.10 (Regularization). Assume that (A1) and (A9) hold. Let $u \in C(\bar{\Omega})$ be a subsolution of $(\mathrm{N})$, and $u_{n}, \Omega_{n}$ be the functions and sets given by Lemma 3.9 for any $n \in \mathbb{N}$. Define the functions $u_{n}^{m}: \mathbb{R}^{N} \rightarrow \mathbb{R}$ by $u_{n}^{m}(x):=u_{n} * \rho_{m}(x)$, where * denotes the convolution, $\rho_{m}(x):=m \rho(m x)$ for $m \in \mathbb{N}$ and $\rho \in C^{\infty}\left(\mathbb{R}^{n}\right)$ is a standard mollification kernel, i.e., $\rho \geq 0, \operatorname{supp} \rho \subset B(0,1)$ and $\int_{\mathbb{R}^{N}} \rho(x) d x=1$. Then $u_{n}^{m} \in C^{1}(\bar{\Omega})$ and $u_{n}^{m}$ satisfy

$$
\left\|D u_{n}^{m}(x)\right\| \leq h(x)+\omega_{n}\left(\frac{1}{m}\right) \quad \text { for all } x \in \Omega
$$

for all $n, m \in \mathbb{N}$ such that $1 / m \leq \operatorname{dist}\left(\partial \Omega_{2 n}, \Omega_{n}\right)$ and $\omega_{n}:[0, \infty) \rightarrow[0, \infty)$ is a modulus for a fixed $n \in \mathbb{N}$.

Proof. Let $\omega_{n}$ be a modulus of continuity of $h$ on $\bar{\Omega}_{2 n}$. We calculate that for all $x \in \Omega_{n}$,

$$
\begin{aligned}
0 & \geq \rho_{m} *\left(\left\|D u_{n}(\cdot)\right\|-h(\cdot)\right)(x) \\
& =\int_{B\left(x, \frac{1}{m}\right)} \rho_{m}(x-y)\left(\left\|D u_{n}(y)\right\|-h(y)\right) d y \\
& \geq \int_{B\left(x, \frac{1}{m}\right)} \rho_{m}(x-y)\left(\left\|D u_{n}(y)\right\|-h(x)-\omega_{n}\left(\frac{1}{m}\right)\right) d y \\
& \geq\left\|\rho_{m} * D u_{n}(x)\right\|-h(x)-\omega_{n}\left(\frac{1}{m}\right) \\
& =\left\|D u_{n}^{m}(x)\right\|-h(x)-\omega_{n}\left(\frac{1}{m}\right) .
\end{aligned}
$$

We have used Jensen's inequality in the third inequality.

By Lemma 3.9 we have

$$
\left\|D u_{n}(x)\right\| \leq n \quad \text { for almost every } x \in \Omega .
$$

Therefore, we have

$$
\left\|D v_{n}^{m}(x)\right\| \leq \int_{\mathbb{R}^{N}} \rho_{m}(x-y)\left\|D v_{n}(y)\right\| d y \leq n \int_{\mathbb{R}^{N}} \rho_{m}(x-y) d y=n
$$

for any $x \in \Omega \backslash \Omega_{n}$, which implies

$$
\left\|D u_{n}^{m}(x)\right\|-h(x) \leq n-n=0 \quad \text { for all } x \in \Omega \backslash \Omega_{n} .
$$


Proof of Theorem 3.8. For a small constant $\alpha>0$, there exists $r_{\alpha}>0$ such that

$$
u(x) \leq v(x)+\alpha \quad \text { on } \mathcal{A}_{\alpha}:=\left\{x+y \mid x \in \mathcal{A}_{h}, y \in \bar{B}\left(0, r_{\alpha}\right)\right\} .
$$

Set $v_{\alpha}(x):=v(x)+\alpha$ for $x \in \bar{\Omega}$. We prove that

$$
u(x) \leq v_{\alpha}(x) \text { for all } x \in \bar{\Omega} \backslash \mathcal{A}_{\alpha}^{i},
$$

where $\mathcal{A}_{\alpha}^{i}$ is the set of interior points of $\mathcal{A}_{\alpha}$. Suppose that

$$
\max _{\bar{\Omega} \backslash \mathcal{A}_{\alpha}^{i}}\left(u-v_{\alpha}\right) \geq 5 \theta>0 \text { for some } \theta>0 .
$$

For suitable large $n, m \in \mathbb{N}$ such that $1 / m \leq \operatorname{dist}\left(\partial \Omega_{2 n}, \Omega_{n}\right)$, we may assume that

$$
\max _{\bar{\Omega} \backslash \mathcal{A}_{\alpha}^{i}}\left(u_{n}^{m}-v_{\alpha}\right) \geq 4 \theta
$$

and

$$
u_{n}^{m}(x)-v_{\alpha}(x) \leq \theta \quad \text { for all } x \in \mathcal{A}_{\alpha},
$$

where $u_{n}^{m}$ are the functions in Lemma 3.10. Fix such an $n \in \mathbb{N}$ and we shall send $m \rightarrow \infty$ later.

For $\lambda \in(0,1)$, we set

$$
u_{\lambda}(x):=\lambda u_{n}^{m}(x) \text { for all } x \in \bar{\Omega} .
$$

Fix $\lambda \in(0,1)$ as close to 1 such that

$$
\max _{\bar{\Omega} \backslash \mathcal{A}_{\alpha}^{i}}\left(u_{\lambda}-v_{\alpha}\right) \geq 3 \theta \text { and } u_{\lambda}(x)-v_{\alpha}(x) \leq 2 \theta \text { for all } x \in \mathcal{A}_{\alpha} .
$$

Let $z_{m}(=z(m, n, \lambda)) \in \bar{\Omega} \backslash \mathcal{A}_{\alpha}^{i}$ be a point which satisfies

$$
\left(u_{\lambda}-v_{\alpha}\right)\left(z_{m}\right)=\max _{\bar{\Omega} \backslash \mathcal{A}_{\alpha}^{i}}\left(u_{\lambda}-v_{\alpha}\right) .
$$

Since $u_{\lambda} \in C^{1}(\bar{\Omega})$ and $v_{\alpha}$ is a supersolution, we see that $z_{m} \in \Omega \backslash \mathcal{A}_{\alpha}^{i}$. By the choice of $\lambda$ we have $z_{m} \in \Omega \backslash \mathcal{A}_{\alpha}$. Moreover, we have

$$
\left\|D u_{\lambda}\left(z_{m}\right)\right\| \geq h\left(z_{m}\right) .
$$

We may assume that

$$
z_{m} \rightarrow \bar{z} \in \bar{\Omega} \backslash \mathcal{A}_{\alpha}^{i} \quad \text { as } m \rightarrow \infty
$$

by taking a subsequence if necessary, since $\bar{\Omega} \backslash \mathcal{A}_{\alpha}^{i}$ is a compact set. Suppose that $\bar{z} \in \partial \Omega$. Then we have $h\left(z_{m}\right) \rightarrow+\infty$ as $m \rightarrow \infty$, which contradicts the inequality $\left\|D u_{\lambda}(x)\right\| \leq \lambda n$ for all $x \in \Omega$. Therefore, we have $\bar{z} \in \Omega$. Moreover, we may assume that

$$
\operatorname{dist}\left(z_{m}, \partial \Omega\right) \geq \alpha(n)>0
$$

for all suitable large $m \in \mathbb{N}$ and some constant $\alpha(n)>0$ which is independent of $m$. We also have $z_{m} \notin \partial \mathcal{A}_{\alpha}$ due to (3.9). Thus, we can choose $r(=r(n, \lambda))>0$ which is independent of $m$ such that $B\left(z_{m}, r\right) \subset \Omega^{n} \backslash \mathcal{A}_{\alpha}$ for all $m \in \mathbb{N}$, where $\Omega^{n}:=\{x \in \Omega \mid \operatorname{dist}(x, \partial \Omega)>\alpha(n) / 2\}$.

Let us consider the function

$$
\Phi(x, y):=u_{\lambda}(x)-v_{\alpha}(y)-\frac{|x-y|^{2}}{2 \varepsilon}-\left|y-z_{m}\right|^{2} \quad \text { for all } x, y \in \bar{\Omega} \text { and } \varepsilon>0 .
$$


Let $x_{\varepsilon}, y_{\varepsilon} \in \bar{\Omega}$ be points which satisfy $\Phi\left(x_{\varepsilon}, y_{\varepsilon}\right)=\max _{\bar{\Omega}^{2}} \Phi$. We obtain that $x_{\varepsilon}, y_{\varepsilon} \rightarrow z_{m}$ as $\varepsilon \rightarrow 0$. Indeed, since $\Phi\left(x_{\varepsilon}, y_{\varepsilon}\right) \geq \Phi\left(z_{m}, z_{m}\right)$, we have

$$
\frac{1}{\varepsilon}\left|x_{\varepsilon}-y_{\varepsilon}\right|^{2}+\left|y_{\varepsilon}-z_{m}\right|^{2} \leq u_{\lambda}\left(x_{\varepsilon}\right)-v_{\alpha}\left(y_{\varepsilon}\right)-\left(u_{\lambda}-v_{\alpha}\right)\left(z_{m}\right) .
$$

We may assume that $x_{\varepsilon_{j}}, y_{\varepsilon_{j}} \rightarrow z_{0}$ as $j \rightarrow \infty$ for some $z_{0} \in \bar{\Omega}$ by taking subsequences $\left\{x_{\varepsilon_{j}}\right\}_{j},\left\{y_{\varepsilon_{j}}\right\}_{j}$ if necessary, since $\bar{\Omega}$ is compact. We have

$$
\limsup _{\varepsilon \rightarrow 0}\left(\frac{1}{\varepsilon}\left|x_{\varepsilon}-y_{\varepsilon}\right|^{2}+\left|y_{\varepsilon}-z_{m}\right|^{2}\right) \leq\left(u_{\alpha}-v_{\lambda}\right)\left(z_{0}\right)-\left(u_{\alpha}-v_{\lambda}\right)\left(z_{m}\right) \leq 0 .
$$

Thus, we obtain that $z_{0}=z_{m}$ and we may actually assume that $x_{\varepsilon}, y_{\varepsilon} \in B\left(z_{m}, r\right)$ for suitable small $\varepsilon \in(0,1)$.

The definition of viscosity solutions immediately implies the following inequalities:

$$
\begin{gathered}
\left\|\frac{1}{\lambda \varepsilon}\left(x_{\varepsilon}-y_{\varepsilon}\right)\right\| \leq h\left(x_{\varepsilon}\right)+\omega_{n}\left(\frac{1}{m}\right), \\
\left\|\frac{1}{\varepsilon}\left(x_{\varepsilon}-y_{\varepsilon}\right)-2\left(y_{\varepsilon}-z_{m}\right)\right\| \geq h\left(y_{\varepsilon}\right) .
\end{gathered}
$$

Since $x_{\varepsilon} \in B\left(z_{m}, r\right) \subset \overline{\Omega^{n}}$ for suitable small $\varepsilon>0$, we have

$$
\frac{1}{\lambda}\left\|\frac{1}{\varepsilon}\left(x_{\varepsilon}-y_{\varepsilon}\right)\right\| \leq h\left(x_{\varepsilon}\right)+1 \leq \max _{\overline{\Omega^{n}}} h+1 .
$$

This yields

$$
\frac{1}{\lambda \varepsilon}\left|x_{\varepsilon}-y_{\varepsilon}\right| \leq C_{\lambda, n}
$$

for some constant $C_{\lambda, n}>0$ and we can extract a convergent subsequence $\frac{1}{\varepsilon_{j}}\left(x_{\varepsilon_{j}}-y_{\varepsilon_{j}}\right)$ and denote its limit point $p \in B\left(0, \lambda C_{\lambda, n}\right)$.

Sending $j \rightarrow \infty$ in the inequality (3.11), we get

$$
\frac{1}{\lambda}\|p\| \leq h\left(z_{m}\right)+\omega_{n}\left(\frac{1}{m}\right), \quad\|p\| \geq h\left(z_{m}\right) .
$$

Therefore we obtain

$$
h\left(z_{m}\right) \leq \lambda\left(h\left(z_{m}\right)+\omega_{n}\left(\frac{1}{m}\right)\right) .
$$

Sending $m \rightarrow \infty$ yields

$$
h(\bar{z}) \leq \lambda h(\bar{z}),
$$

which yields a contradiction, since $0<h(\bar{z})<+\infty$.

Corollary 3.11. Assume that (A1), (A5), (A6) and (A9) hold. Let $u \in C(\bar{\Omega})$ be a solution of $(\mathrm{N})$. Then

$$
u(x)=\min \left\{d(x, y)+u(y) \mid y \in \mathcal{A}_{h}\right\} \quad \text { for all } x \in \bar{\Omega} .
$$

Proof. We denote by $w(x)$ the right hand side of (3.12). We note that $w$ is a solution of (N) by Proposition 3.7 (ii). By the definition of $d$, we have $u(x)-u(y) \leq d(x, y)$ for all $x, y \in \bar{\Omega}$. Hence we get $u(x) \leq w(x)$ for all $x \in \bar{\Omega}$. Next, by the definition of $w$, we have $w(x) \leq u(x)$ for all $x \in \mathcal{A}_{h}$. Thus we have $w(x) \leq u(x)$ for all $x \in \bar{\Omega}$ by Theorem 3.8. Therefore we conclude that $u=w$ on $\bar{\Omega}$. 
Remark 3.6. Let us consider the equation

$$
\left|u_{x}\right|^{2}=\tan (|x|) \quad \text { in }\left(-\frac{\pi}{2}, \frac{\pi}{2}\right) \text { with } u(0)=0 ;
$$

we have the multiplicity of solutions. Indeed, setting

$$
\begin{aligned}
& u(x):=\int_{0}^{|x|}(\tan (y))^{1 / 2} d y \text { for all } x \in[-\pi / 2, \pi / 2], \\
& v(x):= \begin{cases}\int_{0}^{x}(\tan (y))^{1 / 2} d y & \text { for all } x \in[0, \pi / 2], \\
-\int_{0}^{-x}(\tan (y))^{1 / 2} d y & \text { for all } x \in[-\pi / 2,0),\end{cases}
\end{aligned}
$$

we see that $\pm u, \pm v$ are solutions of (3.13). But $u$ is the only solution of (3.13) which satisfies the singular Neumann condition defined by Definition 1 .

\section{LARGE-TIME BEHAVIOR OF SOLUTIONS}

Throughout this section we assume the following structural conditions for the equation $(\mathrm{C})$ as well as a regularity condition on initial data.

(B1) The function $m:[0, \infty) \rightarrow[0,1)$ is a strictly increasing Lipschitz function with $m(0)=: m_{0} \in[0,1)$, and $m(r) \rightarrow 1$ as $r \rightarrow \infty$.

(B2) The function $f$ satisfies

$$
\left\{x \in \mathbb{R}^{N} \mid f(x)=0, \sigma(x)=\bar{\sigma}\right\} \neq \emptyset,
$$

where we set $\bar{\sigma}:=\sup \left\{\sigma(x) \mid x \in \mathbb{R}^{N}\right\}$. (See Remark 5.1 for a physical interpretation.)

(B3) The function $u_{0}$ is Lipschitz continuous in $\mathbb{R}^{N}$.

We set

$$
\begin{aligned}
c & :=\sup _{x \in \mathbb{R}^{N}} \min _{p \in \mathbb{R}^{N}}(H(x, p)-f(x))=\bar{\sigma} m_{0}, \\
\Omega_{e} & :=\left\{x \in \mathbb{R}^{N} \mid \sup _{p \in \mathbb{R}^{N}}(H(x, p)-f(x))>c\right\} \\
& =\left\{x \in \mathbb{R}^{N} \mid \sigma(x)-f(x)>c\right\}, \\
\Omega_{d} & :=\left\{x \in \mathbb{R}^{N} \mid \sup _{p \in \mathbb{R}^{N}}(H(x, p)-f(x))<c\right\}, \\
& =\left\{x \in \mathbb{R}^{N} \mid \sigma(x)-f(x)<c\right\}, \\
\mathcal{A} & :=\left\{x \in \mathbb{R}^{N} \mid \min _{p \in \mathbb{R}^{N}}(H(x, p)-f(x))=c\right\} \\
& =\left\{x \in \mathbb{R}^{N} \mid f(x)=0, \sigma(x)=\bar{\sigma}\right\},
\end{aligned}
$$

where $H(x, p)=\sigma(x) m(\|p\|)$. We call the set $\Omega_{e}$ the effective domain for $(\mathrm{C})$ if $\Omega_{e}$ is a domain in $\mathbb{R}^{N}$ and note that $\mathcal{A}$ is a compact subset of $\Omega_{e}$. For any $x \in \Omega_{e}$, there exists $p \in \mathbb{R}^{N}$ such that we have $\sigma(x) m(\|p\|)>f(x)+c$, which implies

$$
1>m(\|p\|)>\frac{f(x)+c}{\sigma(x)} .
$$

Thus we can define the function $h: \Omega_{e} \rightarrow \mathbb{R}$ by

$$
h(x):=m^{-1}\left(\frac{f(x)+c}{\sigma(x)}\right) \text { for all } x \in \Omega_{e} .
$$

Henceforth, we use the above notation. We notice that

$$
\mathcal{A}=\left\{x \in \mathbb{R}^{N} \mid h(x)=0\right\} .
$$


4.1. Large-time asymptotics. We now state the result of the large-time behavior of solutions of $(\mathrm{C})$.

Theorem 4.1. Assume that (B1)-(B3) hold. Let $u \in C\left(\mathbb{R}^{N} \times[0, \infty)\right)$ be the viscosity solution of $(\mathrm{C})$. If the comparison principle taking account of data on $\mathcal{A}$ for

$$
\begin{cases}\|D u\|=h(x) & \text { in } \Omega_{e}, \\ \frac{\partial u}{\partial n}=+\infty & \text { on } \partial \Omega_{e}\end{cases}
$$

holds, then we have

$$
u(x, t)+c t \rightarrow v_{\infty}(x) \quad \text { uniformly on each compact subset of } \Omega_{e}
$$

and

$$
u(x, t)+c t \rightarrow+\infty \quad \text { uniformly on each compact subset of } \Omega_{d}
$$

as $t \rightarrow \infty$ for a solution $v_{\infty} \in C\left(\bar{\Omega}_{e}\right)$ of $(\mathrm{S})$.

If we add the following technical assumptions

(B4) the set $\Omega_{e}$ is a bounded domain and $\Omega_{d}$ is nonempty,

(B5) $h$ satisfies (A7) and (A9),

then we see that the comparison principle taking account of data on $\mathcal{A}$ holds by Theorem 3.8. In Section 4.5 we discuss functions $\sigma, m$ which satisfies the assumptions above.

Remark 4.1. (i) It is worth noticing here that $\Omega_{e}$ and $c$ do not depend on the initial value.

(ii) We set

$$
K_{c}:=\left\{x \in \mathbb{R}^{N} \mid \sup _{p \in \mathbb{R}^{N}}(H(x, p)-f(x))=c\right\} .
$$

We do not know if $u+c t$ converges on $K_{c}$ or not.

(iii) The authors in 21] introduce the new definition (see Definition 2) of solutions to the stationary equation

$$
\sigma(x) m(\|D u\|)=f(x)+c \quad \text { in } \mathbb{R}^{N} .
$$

We present the equivalence between the solutions defined by Definition 1 and Definition 2 precisely in Appendix 5.2.

\subsection{Construction of subsolutions and supersolutions of (C).}

Proposition 4.2. Assume (B1) holds. A subsolution $w^{-} \in C\left(\mathbb{R}^{N} \times[0, \infty)\right)$ and a supersolution $w^{+} \in C\left(\mathbb{R}^{N} \times[0, \infty)\right)$ of $(\mathrm{C})$ exist which satisfy the following:

(i) $\left|w^{ \pm}(x, t)+c t\right| \leq C$ for all $x \in \Omega_{e}, t \in[0, \infty)$ and some $C>0$,

(ii) $w^{ \pm}(x, t)+c t \rightarrow+\infty$ uniformly on each compact subset of $\Omega_{d}$ as $t \rightarrow \infty$.

Proof. We first construct a subsolution. Set

$$
G_{1}(x):=-\sup _{p \in \mathbb{R}^{N}}\{H(x, p)-f(x)\}(=f(x)-\sigma(x)) .
$$

By Theorem 3.2 we may choose a solution $W_{1} \in C\left(\mathbb{R}^{n}\right)$ of $(\mathrm{S})$ such that

$$
\begin{aligned}
& W_{1}(x) \leq u_{0}(x) \text { for all } x \in \mathbb{R}^{N} \backslash \bar{\Omega}_{e}, \\
& W_{1}(x) \text { is a subsolution of }(\mathrm{S}) \text { in } \Omega_{e} .
\end{aligned}
$$


Define the function $w^{-} \in C\left(\mathbb{R}^{N} \times[0, \infty)\right)$ by

$$
w^{-}(x, t):= \begin{cases}W_{1}(x)-c t & \text { for all }(x, t) \in \bar{\Omega}_{e} \times[0, \infty), \\ W_{1}(x)+G_{1}(x) t & \text { for all }(x, t) \in\left(\mathbb{R}^{N} \backslash \bar{\Omega}_{e}\right) \times[0, \infty) .\end{cases}
$$

It is easily seen that $w^{-}$is a subsolution of (1.1) in $\Omega_{e} \times(0, \infty)$. Indeed, let $w^{-}-\phi$ take a maximum at $\left(x_{0}, t_{0}\right) \in \Omega_{e} \times(0, \infty)$ for some $\phi \in C^{1}\left(\mathbb{R}^{N} \times(0, \infty)\right)$. Then we have $\phi_{t}\left(x_{0}, t_{0}\right)=-c$ and

$$
\left\|D \phi\left(x_{0}, t_{0}\right)\right\| \leq h\left(x_{0}\right),
$$

which is equivalent to

$$
\sigma\left(x_{0}\right) m\left(\left\|D \phi\left(x_{0}, t_{0}\right)\right\|\right) \leq f\left(x_{0}\right)+c .
$$

Therefore, we get

$$
\phi_{t}\left(x_{0}, t_{0}\right)+\sigma\left(x_{0}\right) m\left(\left\|D \phi\left(x_{0}, t_{0}\right)\right\|\right) \leq f\left(x_{0}\right) .
$$

Let $w^{-}-\phi$ take a maximum at $\left(x_{0}, t_{0}\right) \in\left(\mathbb{R}^{N} \backslash \Omega_{e}\right) \times(0, T)$ for some $C^{1}\left(\mathbb{R}^{N} \times(0, \infty)\right)$. Noting that $\phi_{t}\left(x_{0}, t_{0}\right)=G_{1}\left(x_{0}\right)$, we have

$$
\begin{aligned}
& \phi_{t}\left(x_{0}, t_{0}\right)+H\left(x_{0}, D \phi\left(x_{0}, t_{0}\right)\right)-f\left(x_{0}\right) \\
= & G_{1}\left(x_{0}\right)+H\left(x_{0}, D \phi\left(x_{0}, t_{0}\right)\right)-f\left(x_{0}\right) \\
= & -\sup _{p \in \mathbb{R}^{N}}\left(H\left(x_{0}, p\right)-f\left(x_{0}\right)\right)+H\left(x_{0}, D \phi\left(x_{0}, t_{0}\right)\right)-f\left(x_{0}\right) \\
\leq & 0 .
\end{aligned}
$$

Since $G_{1}(x)=-c$ on $K_{c}$ and $G_{1}(x)>-c$ for all $x \in \Omega_{d}$, we see that $w^{-}$satisfies (i)-(ii).

Similarly, we can construct a supersolution. Set

$$
G_{2}(x):=-\inf _{p \in \mathbb{R}^{N}}\{H(x, p)-f(x)\}\left(=f(x)-\sigma(x) m_{0}\right)
$$

and choose a function $W_{2} \in C\left(\mathbb{R}^{N}\right)$ such that

$$
\begin{aligned}
& W_{2}(x) \geq u_{0}(x) \text { for all } x \in \mathbb{R}^{N} \backslash \bar{\Omega}_{e}, \\
& W_{2}(x) \text { is a supersolution of }(\mathrm{S}) .
\end{aligned}
$$

Define the function $w^{+} \in C\left(\mathbb{R}^{N} \times[0, \infty)\right)$ by

$$
w^{+}(x, t):= \begin{cases}W_{2}(x)-c t & \text { for all }(x, t) \in \bar{\Omega}_{e} \times[0, \infty), \\ W_{2}(x)+G_{2}(x) t & \text { for all }(x, t) \in\left(\mathbb{R}^{N} \backslash \bar{\Omega}_{e}\right) \times[0, \infty) .\end{cases}
$$

Then we see that $w^{+}$is a supersolution of (1.1) which satisfies (i)-(ii).

4.3. Stability and Proof of Theorem 4.1. We define the functions $u^{+}, u^{-}$: $\mathbb{R}^{N} \rightarrow \mathbb{R} \cup\{+\infty\}$ by

$$
\begin{aligned}
& u^{+}(x):=\limsup _{t \rightarrow \infty} *(u(x, t)+c t)=\lim _{t \rightarrow \infty} \sup \{u(y, s)+c s|s \geq t,| x-y \mid \leq 1 / t\}, \\
& u^{-}(x):=\liminf _{t \rightarrow \infty}(u(x, t)+c t)=\lim _{t \rightarrow \infty} \inf \{u(y, s)+c s|s \geq t,| x-y \mid \leq 1 / t\} .
\end{aligned}
$$

Since $m$ is Lipschitz continuous, we have

$$
|H(x, p)-H(x, q)| \leq C(|x|+1)|p-q|
$$


for all $x \in \mathbb{R}^{N}, p, q \in \mathbb{R}^{N}$, and some $C>0$. Therefore, the comparison principle for unbounded viscosity solutions of $(\mathrm{C})$ holds (see [27, Theorem 2.5] or [1, Theorem 3.6]). By Proposition 4.2 we have $u^{+} \in \operatorname{USC}\left(\Omega_{e} \cup K_{c}, \mathbb{R}\right), u^{-} \in \operatorname{LSC}\left(\Omega_{e} \cup K_{c}, \mathbb{R}\right)$ and

$$
u^{+}(x)=+\infty \text { and } u^{-}(x)=+\infty \text { for all } x \in \Omega_{d} .
$$

By the standard stability theorem in the theory of viscosity solution, we see that $u^{+}$satisfies

$$
H\left(x, D u^{+}(x)\right) \leq f(x) \text { in } \Omega_{e}
$$

in the viscosity sense, which implies

$$
\left\|D u^{+}(x)\right\| \leq h(x) \quad \text { in } \Omega_{e} .
$$

In view of Lemma 3.3, the function $u^{+}$is uniformly continuous in $\Omega_{e}$, and therefore $u^{+}$can be extended uniquely to the function on $\bar{\Omega}_{e}$ by continuity. We denote this function by $v^{+} \in C\left(\bar{\Omega}_{e}\right)$, i.e., we set

$$
v^{+}(x):= \begin{cases}u^{+}(x) & \text { for all } x \in \Omega_{e}, \\ \lim _{y \rightarrow x, y \in \Omega_{e}} u^{+}(y) & \text { for all } x \in \partial \Omega_{e} .\end{cases}
$$

Remark 4.2. We do not know whether $u^{+}=v^{+}$on $K_{c}$ or not.

Proposition 4.3 (Stability on half-relaxed limits). Assume that (B1) holds. The functions $v^{+}$and $u^{-}$are a subsolution and a supersolution of $(\mathrm{S})$, respectively.

Proof. We only need to prove that $u^{-}$satisfies the boundary condition defined by Definition 1. Let $\varepsilon>0$ and set $u^{\varepsilon}(x, t):=u(x, t / \varepsilon)+c t / \varepsilon$. Then it is clear that $u^{\varepsilon}$ satisfies

$$
\varepsilon u_{t}^{\varepsilon}+H\left(x, D u^{\varepsilon}\right)=f(x)+c \text { in } \mathbb{R}^{N} \times(0, \infty)
$$

in the viscosity sense. Set

$$
\underline{u}(x, t):=\left(\liminf _{\varepsilon \rightarrow 0} u^{\varepsilon}\right)(x, t)
$$

and then we have $\underline{u}(x, t)=u^{-}(x)$ for all $(x, t) \in \bar{\Omega}_{e} \times(0, \infty)$.

Suppose that $u^{-}-\phi$ takes a strict minimum over $\bar{\Omega}_{e}$ at some point $x_{0} \in \partial \Omega_{e}$ and some function $\phi \in C^{1}\left(\bar{\Omega}_{e}\right)$. Then there exists a function $\psi \in C^{1}\left(\mathbb{R}^{N} \times(0, \infty)\right)$ such that $\underline{u}-\psi$ takes a strict minimum at $\left(x_{0}, t_{0}\right) \in \partial \Omega_{e} \times(0, \infty)$ for some $t_{0}>0$.

By standard arguments, there exist $\left\{\varepsilon_{n}\right\}_{n \in \mathbb{N}} \subset(0,1)$ and $\left\{\left(x_{n}, t_{n}\right)\right\}_{n \in \mathbb{N}} \subset \bar{\Omega}_{e} \times$ $(0, \infty)$ such that

$$
\begin{gathered}
\varepsilon_{n} \rightarrow 0, \quad\left(x_{n}, t_{n}\right) \rightarrow\left(x_{0}, t_{0}\right), \\
u^{\varepsilon_{n}}\left(x_{n}, t_{n}\right) \rightarrow \underline{u}\left(x_{0}, t_{0}\right) \quad \text { as } n \rightarrow+\infty
\end{gathered}
$$

and

Then we have

$$
\left(u^{\varepsilon_{n}}-\psi\right)\left(x_{n}, t_{n}\right)=\min _{\mathbb{R}^{N} \times(0, \infty)}\left(u^{\varepsilon_{n}}-\psi\right) .
$$

$$
\varepsilon_{n} \psi_{t}\left(x_{n}, t_{n}\right)+H\left(x_{n}, D \psi\left(x_{n}, t_{n}\right)\right) \geq f\left(x_{n}\right)+c .
$$

Sending $n \rightarrow \infty$ yields

$$
H\left(x_{0}, D \psi\left(x_{0}, t_{0}\right)\right) \geq f\left(x_{0}\right)+c=\sigma\left(x_{0}\right) .
$$

Since $\left|D \psi\left(x_{0}, t_{0}\right)\right|<+\infty$ and $m(\|p\|)<1$ for all $p \in \mathbb{R}^{N}$, we see that $\sigma\left(x_{0}\right)>$ $\sigma\left(x_{0}\right) m\left(\left\|D \psi\left(x_{0}, t_{0}\right)\right\|\right)=H\left(x_{0}, D \psi\left(x_{0}, t_{0}\right)\right)$, which implies a contradiction. 
Lemma 4.4 (Monotonicity on $\mathcal{A}$ ). Assume that (B1), (B2) hold. For any $x \in \mathcal{A}$, the function $t \mapsto u(x, t)+$ ct is nonincreasing in $(0, \infty)$.

Remark 4.3. By (B1) we have

$$
H(x, p)-f(x) \geq H(x, 0)-f(x) \text { and } \max _{x \in \mathbb{R}^{N}}(H(x, 0)-f(x))=c,
$$

which is essentially the same as (1.12). In [39, Lemma 2.4] it is proved that the same monotonicity results under the assumption that $u$ is Lipschitz continuous on $\mathcal{M} \times[0, \infty)$, where $\mathcal{M}$ is a smooth compact $N$-dimensional manifold without boundary. It is worthwhile to mention that in the proof of the above lemma we do not use any regularity of $u$.

Proof. We prove that

$$
u(x, t+\delta)+c(t+\delta) \leq u(x, t)+c t \quad \text { for all } x \in \mathcal{A}, t, \delta>0 .
$$

We argue by contradiction. Suppose that there would exist $x_{0} \in \mathcal{A}, t_{0}>0$ and $\delta_{0}>0$ such that $u\left(x_{0}, t_{0}+\delta_{0}\right)+c\left(t_{0}+\delta_{0}\right)>u\left(x_{0}, t_{0}\right)+c t_{0}$.

Choose $r>0$ and $\delta^{\prime} \in\left(0, \delta_{0}\right)$ such that

$$
u\left(x, t_{0}+\delta^{\prime}\right)+c\left(t_{0}+\delta^{\prime}\right)>u\left(x, t_{0}\right)+c t_{0} \text { for all } x \in \bar{B}\left(x_{0}, r\right) .
$$

Take $\varepsilon>0$ so small that we have

(4.9) $u\left(x, t_{0}\right)+c t_{0}-\frac{\varepsilon}{\delta_{0}}<u\left(x, t_{0}+\delta^{\prime}\right)+c\left(t_{0}+\delta^{\prime}\right)-\frac{\varepsilon}{\delta_{0}-\delta^{\prime}} \quad$ for all $x \in \bar{B}\left(x_{0}, r\right)$.

For any $\alpha>0$, we define the function $\Phi: B\left(x_{0}, r\right) \times\left(t_{0}, t_{0}+\delta_{0}\right) \rightarrow \mathbb{R}$ by

$$
\Phi(x, t):=u(x, t)+c t-\frac{\alpha\left|x-x_{0}\right|^{2}}{r^{2}-\left|x-x_{0}\right|^{2}}-\frac{\left|x-x_{0}\right|^{2}}{\alpha}-\frac{\varepsilon}{t_{0}+\delta_{0}-t} .
$$

Note that

$$
\begin{gathered}
\Phi\left(x, t_{0}\right)<\Phi\left(x, t_{0}+\delta^{\prime}\right) \quad \text { for all } x \in B\left(x_{0}, r\right) \text { in view of (4.9), } \\
\Phi(x, t) \rightarrow-\infty \quad \text { as } t \rightarrow t_{0}+\delta_{0} \text { for all } x \in B\left(x_{0}, r\right), \\
\Phi(x, t) \rightarrow-\infty \quad \text { as }\left|x-x_{0}\right| \rightarrow r \text { for all } s \in\left(t_{0}, t_{0}+\delta_{0}\right) .
\end{gathered}
$$

Their properties imply that there exists an interior point $\left(x_{\alpha}, t_{\alpha}\right) \in B\left(x_{0}, r\right) \times$ $\left(t_{0}, t_{0}+\delta_{0}\right)$ such that

$$
\Phi\left(x_{\alpha}, t_{\alpha}\right)=\max _{B\left(x_{0}, r_{0}\right) \times\left[t_{0}, t_{0}+\delta_{0}\right)} \Phi(x, t) .
$$

Since $\Phi\left(x_{\alpha}, t_{\alpha}\right) \geq \Phi\left(x_{0}, t_{0}\right)$ and $u+c t$ is bounded on $\mathcal{A} \times[0, \infty)$, we observe that

$$
\begin{aligned}
& \frac{\alpha\left|x_{\alpha}-x_{0}\right|^{2}}{r^{2}-\left|x_{\alpha}-x_{0}\right|}+\frac{\left|x_{\alpha}-x_{0}\right|^{2}}{\alpha}+\frac{\varepsilon}{t_{0}+\delta_{0}-t_{\alpha}} \\
\leq & u\left(x_{\alpha}, t_{\alpha}\right)+c t_{\alpha}-\left(u\left(x_{0}, t_{0}\right)+c t_{0}\right)+\frac{\varepsilon}{t_{0}} \leq C
\end{aligned}
$$

and we have $x_{\alpha} \rightarrow x_{0}$ as $\alpha \rightarrow 0$. We also may assume $t_{\alpha} \rightarrow \bar{t}$ as $\alpha \rightarrow 0$ for some $\bar{t}(=\bar{t}(\varepsilon)) \in\left[t_{0}, t_{0}+\delta_{0}\right)$ by taking a subsequence if necessary.

Thus, the definition of viscosity solutions immediately implies the following inequality:

$$
\begin{aligned}
\frac{\varepsilon}{\left(t_{0}+\delta_{0}-t_{\alpha}\right)^{2}}-c & \leq-H\left(x_{\alpha}, \frac{-2 \alpha r^{2}\left(x_{\alpha}-x_{0}\right)}{\left(\left|x_{\alpha}-x_{0}\right|^{2}-r^{2}\right)^{2}}+\frac{2\left(x_{\alpha}-x_{0}\right)}{\alpha}\right)+f\left(x_{\alpha}\right) \\
& \leq-\sigma\left(x_{\alpha}\right) m_{0}+f\left(x_{\alpha}\right) .
\end{aligned}
$$


Sending $\alpha \rightarrow 0$, we get

$$
\frac{\varepsilon}{\left(t_{0}+\delta_{0}-\bar{t}\right)^{2}}-c \leq-\sigma\left(x_{0}\right) m_{0}+f\left(x_{0}\right)=-c
$$

which is a contradiction.

Lemma 4.5 (Uniform continuity of solution of (C)). Assume that (B1)-(B3) hold. There exists a constant $r>0$ such that the solution $u$ of $(\mathrm{C})$ is uniformly continuous on $\mathcal{A}_{r} \times[0, \infty)$, where $\mathcal{A}_{r}:=\{x+y \mid x \in \mathcal{A}, y \in B(0, r)\}$.

Proof. We first assume that $u_{0} \in C^{1}\left(\mathbb{R}^{N}\right) \cap \operatorname{Lip}\left(\mathbb{R}^{N}\right)$ and $f$ is bounded. We set

$$
\begin{aligned}
& \bar{m}:=\sup _{x \in \mathbb{R}^{N}} m\left(\left\|D u_{0}(x)\right\|\right), \\
& v^{-}(x, t):=u_{0}(x)-\bar{\sigma} \bar{m} t .
\end{aligned}
$$

We note that $\bar{m} \supsetneqq 1$. We can easily check that $v^{-}$is a subsolution of $(\mathrm{C})$. By the comparison principle for $(\mathrm{C})$ we have $u_{0}(x)-u(x, t) \leq \bar{\sigma} \bar{m} t$, and moreover by the maximum principle we obtain $u(x, s)-u(x, t+s) \leq \bar{\sigma} \bar{m} t$ for all $x \in \mathbb{R}^{N}$ and $t, s \geq 0$. Thus, we get

$$
u_{t}(x, t) \geq-\bar{\sigma} \bar{m} \text { in }(0, \infty) \text { in the viscosity sense }
$$

for all $x \in \mathbb{R}^{N}$ and therefore

$$
\sigma(x) m(\|D u(\cdot, t)\|) \leq f(x)+\bar{\sigma} \bar{m} \text { in } \mathbb{R}^{N}
$$

for all $t \in(0, \infty)$.

Since $\sigma(x)=\bar{\sigma}, f(x)=0$ for all $x \in \mathcal{A}$ and $\bar{m}<1$, there exists a constant $r>0$ such that

$$
\sigma(x)>f(x)+\bar{\sigma} \bar{m}
$$

for all $x \in \mathcal{A}_{r}$. Therefore we see that

$$
|D u(x, t)| \leq C_{1}\|D u(x, t)\| \leq C_{1} m^{-1}\left(\frac{f(x)+\bar{\sigma} \bar{m}}{\sigma(x)}\right) \leq C_{2} \text { in } \mathcal{A}_{r}
$$

in the viscosity sense for some $C_{1}, C_{2}>0$ and all $t \in[0, \infty)$, which implies $\{u(\cdot, t)\}_{t \geq 0}$ is equicontinuous on $\mathcal{A}_{r}$. We also see that $v^{+}(x, t):=u_{0}(x)+C_{f} t$ is a supersolution of $(\mathrm{C})$, where $C_{f}:=\sup f$ and therefore we have $|u(x, t+s)-u(x, s)| \leq$ $\bar{\sigma} \bar{m} \vee C_{f} t$ for all $x \in \mathbb{R}^{N}$ and $t, s \geq 0$. Thus, we see that $u \in \operatorname{UC}\left(\mathcal{A}_{r} \times[0, \infty)\right)$.

We finally remove the regularity of assumption on $u_{0}$ and the boundedness of $f$. We can choose a sequence of functions $\left\{u_{0}^{k}\right\}_{k \in \mathbb{N}} \subset C^{1}\left(\mathbb{R}^{N}\right) \cap \operatorname{Lip}\left(\mathbb{R}^{N}\right)$ such that $u_{0}^{k}$ converges locally uniformly to $u_{0}$ as $k \rightarrow \infty$ and $\left\{f^{k}\right\}_{k \in \mathbb{N}} \subset C\left(\mathbb{R}^{N}\right)$ such that $f^{k}$ are bounded, $\left\{f^{k}(\cdot)=0\right\}=\{f(\cdot)=0\}$ for all $k \in \mathbb{N}$ and $f^{k}$ converges locally uniformly to $f$ as $k \rightarrow \infty$. By the maximum principle we see that the solutions $u^{k} \in \mathrm{UC}\left(\mathcal{A}_{r} \times[0, \infty)\right)$ of $(\mathrm{C})$ with $u_{0}=u_{0}^{k}$ converges locally uniformly to $u$ as $k \rightarrow \infty$. We note that we can choose such an $r$ that is independent of $k$, since we are assuming $u_{0} \in \operatorname{Lip}\left(\mathbb{R}^{N}\right)$. From this observation we see that $u \in \mathrm{UC}\left(\mathcal{A}_{r} \times[0, \infty)\right)$.

Corollary 4.6 (Convergence on $\mathcal{A})$. Assume that (B1)-(B3) hold. The function $u(x, t)+c t$ converges uniformly on $\mathcal{A}$ as $t \rightarrow \infty$. Moreover, we have

$$
v^{+}(x)=u^{-}(x) \text { for all } x \in \mathcal{A} \text {. }
$$


Proof. By Lemma 4.5 we see that $v^{+}\left(=u^{+}\right), u^{-} \in C(\mathcal{A})$. In view of Dini's theorem we see that $u+c t$ converges uniformly on $\mathcal{A}$ as $t \rightarrow \infty$, and by a property of half relaxed limit we obtain $v^{+}=u^{+}=u^{-}$on $\mathcal{A}$.

Proof of Theorem 4.1. A straightforward consequence of Proposition 4.3. Corollary 4.6 and the comparison principle for (S) is $v^{+}=u^{-}$on $\bar{\Omega}_{e}$. (Notice that if we assume that (B4)-(B5) hold, then Theorem 3.8 holds.) Thus, we get

$$
u^{+}(x)=u^{-}(x)=: v_{\infty}(x) \text { for all } x \in \Omega_{e},
$$

which implies that

$$
u(x, t)+c t \rightarrow v_{\infty}(x) \quad \text { uniformly on each compact subset of } \Omega_{e} \text { as } t \rightarrow \infty
$$

for a solution $v_{\infty} \in C\left(\bar{\Omega}_{e}\right)$ of (S). It is clear to see that

$$
u(x, t)+c t \rightarrow+\infty \quad \text { uniformly on each compact subset of } \Omega_{d}
$$

as $t \rightarrow \infty$ from Proposition 4.2 .

Remark 4.4. For generalization, it is worthwhile saying that if we can prove the comparison principle for $(\mathrm{N})$, then we obtain a similar convergence result on the viscosity solution of

$$
\begin{cases}u_{t}+\sigma(x) m(F(x, D u))=f(x) & \text { in } \mathbb{R}^{N} \times(0, \infty), \\ u(\cdot, 0)=u_{0} & \text { in } \mathbb{R}^{N},\end{cases}
$$

where the function $F$ is assumed to satisfy (A1)-(A7) and the functions $\sigma, m, f, u_{0}$ are assumed to satisfy (B1)-(B5).

4.4. Asymptotic profile on the effective domain $\Omega_{\mathrm{e}}$. We define the functions $\phi_{-}, \phi_{\infty} \in C\left(\bar{\Omega}_{e}\right)$ by

$$
\begin{aligned}
\phi_{-}(x) & :=\inf _{t \geq 0}(u(x, t)+c t), \\
\phi_{\infty}(x) & :=\min \left\{d(x, y)+\phi_{-}(y) \mid y \in \mathcal{A}\right\}, \\
d(x, y):=\sup \{v(x) & -v(y) \mid v \text { is a viscosity subsolution of (4.6) }\} .
\end{aligned}
$$

Note that in view of Theorem 3.4 and Proposition 3.7 (ii), we see that $\phi_{\infty}$ is a solution of $(\mathrm{S})$.

Theorem 4.7 (Asymptotic profile). We have

$$
\phi_{\infty}(x)=\lim _{t \rightarrow \infty}(u(x, t)+c t) \quad \text { for all } x \in \Omega_{e} .
$$

Proof. We write $u_{\infty}(x)$ for the right hand side of (4.11) for $x \in \Omega_{e}$. By the definition of $\phi_{-}$we have $\phi_{-}(x) \leq u(x, t)+c t$ for all $(x, t) \in \bar{\Omega}_{e} \times[0, \infty)$, which implies that $\phi_{-} \leq u_{\infty}$ in $\Omega_{e}$. By the definition of $\phi_{\infty}$ we have $\phi_{\infty} \leq \phi_{-}$on $\mathcal{A}$. Therefore, we have $\phi_{\infty} \leq u_{\infty}$ on $\mathcal{A}$. By Theorem 3.8 we get $\phi_{\infty} \leq u_{\infty}$ on $\bar{\Omega}_{e}$.

In order to see the other inequality, set $\underline{u}(x, t):=\inf _{s \geq t}(u(x, s)+c s)$ and then we can easily see $\underline{u}(x, 0)=\phi_{-}(x)$. Noting that $\phi_{\infty}=\phi_{-}$on $\mathcal{A}$, by the comparison theorem for (S) we have $\phi_{\infty} \geq \phi_{-}=\underline{u}(\cdot, 0)$ on $\Omega_{e}$. We extend $\phi_{\infty}$ to the function on $\mathbb{R}^{n}$ by setting $\phi_{\infty}(x):=+\infty$ for $x \in \mathbb{R}^{n} \backslash \Omega_{e}$. Then we can consider $\phi_{\infty}$ as a solution of (1.1) with $u_{0}=\phi_{\infty} \geq \underline{u}(x, 0)$. Therefore by the comparison principle for $(\mathrm{C})$ we get $\underline{u}(x, t) \leq \phi_{\infty}(x)$ for all $x \in \mathbb{R}^{n}$ and $t \geq 0$. Sending $t \rightarrow 0$ yields $u_{\infty} \leq \phi_{\infty}$ on $\Omega_{e}$. 
4.5. A model describing growing faceted crystals. In this subsection we consider the equation for a model of a growing faceted crystal as explained in the Introduction (see also [9, 12, 44]), and for the reader's convenience we give a simple form of our asymptotic result which is by no means optimal. We consider

$$
u_{t}+\sigma(x) m(\|D u\|)=0 \quad \text { in } \mathbb{R}^{N} \times(0, \infty),
$$

where $m \in C([0, \infty),(0,1))$ which satisfies $(\mathrm{B} 1)$ and

$$
m(0)=m_{0} \in(0,1) \text { and } 1-m(r) \leq e^{-r} \text { for large } r>0,
$$

and $\sigma \in C^{1}\left(\mathbb{R}^{N},(0, \infty)\right)$ is a function which attains the maximum $\bar{\sigma}$ and satisfies $\sigma(x)<\bar{\sigma} m_{0}$ for any $x \in \mathbb{R}^{N} \backslash B(0, r)$ and a suitable large $r>0$. In this case we have

$$
\begin{aligned}
& c=\bar{\sigma} m_{0}, \\
& \Omega_{e}=\left\{x \in \mathbb{R}^{N} \mid \sigma(x)>c\right\}, \\
& \Omega_{d}=\left\{x \in \mathbb{R}^{N} \mid \sigma(x)<c\right\} \text { and } \\
& \mathcal{A}:=\left\{x \in \mathbb{R}^{N} \mid \sigma(x)=\bar{\sigma}\right\} .
\end{aligned}
$$

We set

$$
h(x):=m^{-1}\left(\frac{c}{\sigma(x)}\right) .
$$

Corollary 4.8. Let us assume $m$ satisfies (B1), (4.13) and that $\sigma \in C^{1}\left(\mathbb{R}^{N},(0, \infty)\right)$ satisfies

$$
\Omega_{e}^{\alpha}:=\{\sigma(\cdot)>c+\alpha\} \text { are bounded and convex for all } \alpha \in\left(0, \alpha_{0}\right]
$$

for a small $\alpha_{0}>0$ and

$$
D \sigma(x) \neq 0 \text { on } \partial \Omega_{e} \text {. }
$$

Let $u$ be a solution of (4.12) with $u(\cdot, 0)=u_{0} \in \operatorname{Lip}\left(\mathbb{R}^{N}\right)$ and then we have the result of large-time asymptotics given by Theorems 4.1, 4.7, i.e.,

$$
u(\cdot, t)+c t \rightarrow \phi_{\infty} \quad \text { uniformly on each compact subset of } \Omega_{e}
$$

and

$$
u(\cdot, t)+c t \rightarrow+\infty \quad \text { uniformly on each compact subset of } \Omega_{d}
$$

as $t \rightarrow+\infty$, where $\phi_{\infty}$ is the function given by (4.10).

Proof. We only need to check that $h$ satisfies (A7). We first notice that by (4.13) we have

$$
\begin{aligned}
h(x)=m^{-1}\left(\frac{c}{\sigma(x)}\right) & =m^{-1}\left(1-e^{\log \left(\frac{\sigma(x)-c}{\sigma(x)}\right)}\right) \\
& \leq-\log \left(\frac{\sigma(x)-c}{\sigma(x)}\right) .
\end{aligned}
$$

We only prove that $h$ satisfies (A7) in $\left\{\sigma(\cdot)>c-\alpha_{0}\right\}$. Otherwise it is easy to see that $h$ satisfies (A7), since $h$ is bounded on $\mathbb{R}^{N} \backslash\left\{\sigma(\cdot)>c-\alpha_{0}\right\}$. Fix any $x_{c} \in \partial \Omega_{e}$ 
and then for any $x \in\left\{\sigma(\cdot)>c-\alpha_{0}\right\}$ we have

$$
\begin{aligned}
& \int_{0}^{1} h\left(s x+(1-s) x_{c}\right)\left|x-x_{c}\right| d s \\
\leq & \left|x-x_{c}\right| \int_{0}^{1}\left[-\log \left(\frac{s D \sigma\left(x_{c}\right) \cdot\left(x-x_{c}\right)+o\left(s\left|x-x_{c}\right|\right)}{\sigma\left(s x+(1-s) x_{c}\right)}\right)\right] d s \\
\leq & C\left|x-x_{c}\right||\log | x-x_{c}||
\end{aligned}
$$

for some $C>0$, where $o:[0, \infty) \rightarrow[0, \infty)$ is a function which satisfies $o(r) / r \rightarrow 0$ as $r \rightarrow 0$. Therefore setting $\omega(r):=C r|\log (r)|$ for $r>0$, then we see that $h$ satisfies (A7).

We give an example for which we can calculate the function $\phi_{\infty}$ given by (4.10) concretely. Let $n=1$ and let us set $u_{0} \equiv 0, \sigma(x)=\bar{\sigma}\left(1-x^{2}\right)_{+}$and $m(\|\cdot\|)=m(|\cdot|)$ as considered in 44, where $r_{+}:=\max \{r, 0\}$ for $r \in \mathbb{R}$. Then we have $c=\bar{\sigma} m_{0}$, $\Omega_{e}=\left(-\sqrt{1-m_{0}}, \sqrt{1-m_{0}}\right), \mathcal{A}=\{0\}$ and $h(x)=m^{-1}\left(m_{0} /\left(1-x^{2}\right)_{+}\right)$. Note that 0 is a subsolution of $(\mathrm{C})$ at this case and therefore we have

$$
0 \leq u \leq u+c t \text { on } \mathbb{R}^{N} \times[0, \infty),
$$

which implies $\phi_{-}(x)=\inf _{t \geq 0}(u(x, t)+c t)=0$. Therefore we have $\phi_{\infty}(x)=$ $\min _{y \in \mathcal{A}}\left\{d(x, y)+\phi_{-}(y)\right\}=d(x, 0)$. Moreover, noting that $L(x, \xi)=0$ if $|\xi| \leq 1$ and $L(x, \xi)=+\infty$ if $|\xi|>1$ for all $x \in \Omega_{e}$, by (3.4) we have for any $x \in \Omega_{e}$

$$
\begin{aligned}
d(x, 0) & =\inf \left\{\int_{0}^{t} h(\gamma(s)) d s|t>0, \gamma \in \mathcal{C}(x, t ; 0,0),| \dot{\gamma}(s) \mid \leq 1\right\} \\
& =\int_{0}^{x} h(s) d s=\int_{0}^{x} m^{-1}\left(\frac{m_{0}}{\left(1-s^{2}\right)_{+}}\right) d s .
\end{aligned}
$$

Thus we obtain

$$
\phi_{\infty}(x)=\int_{0}^{x} m^{-1}\left(\frac{m_{0}}{\left(1-s^{2}\right)_{+}}\right) d s \text { for all } x \in \bar{\Omega}_{e} .
$$

Finally we note that in the case where $c=\bar{\sigma} m_{0}<\sigma(x)<\bar{\sigma}$ for all $x \in \mathbb{R}^{N}$, we have $\Omega_{e}=\mathbb{R}^{N}$. In this case the large-time asymptotic of solutions of (C) is similar to that of solutions of coercive HJ equations. More precisely, if we assume the periodicity of $\sigma$ and $u_{0}$, i.e.,

$$
\sigma\left(x+e_{i}\right)=\sigma(x), u_{0}\left(x+e_{i}\right)=u_{0}(x)
$$

for all $x \in \mathbb{R}^{N}$ and $i \in\{1, \ldots, N\}$, where $\left\{e_{i}\right\}_{i}$ is the canonical basis of $\mathbb{R}^{N}$, then any solution $u$ of (4.12) has the large-time asymptotic

$$
u(x, t)+c t \rightarrow v(x) \quad \text { uniformly for } x \in \mathbb{R}^{N}
$$

as $t \rightarrow \infty$, where $v \in C\left(\mathbb{R}^{N}\right)$ is a viscosity solution of

$$
\begin{aligned}
& \sigma(x) m(\|D u\|)=c \quad \text { in } \mathbb{R}^{N}, \\
& u\left(x+e_{i}\right)=u(x) \text { for all } x \in \mathbb{R}^{N}, i \in\{1, \ldots, N\} .
\end{aligned}
$$

We give a sketch of the proof here. Since there exists a periodic viscosity solution of (4.14) in $C\left(\mathbb{R}^{N}\right)$, we see that $u+c t$ is bounded in $\mathbb{R}^{N} \times[0, \infty)$. Therefore we can define the functions $u^{+}(x)=\lim \sup _{t \rightarrow \infty}^{*}(u+c t)$ and $u^{-}(x)=\liminf _{t \rightarrow \infty *}(u+c t)$ for all $x \in \mathbb{R}^{N}$ and moreover we see that $u^{+}=u^{-}$on $\mathcal{A}$. By a comparison principle (see [18, Theorem 6.7]) we obtain $u^{+}=u^{-}$in $\mathbb{R}^{N}$. 
In the theory of crystal growth, it is known that as long as the nonuniformity in supersaturation on the facet is not too large, it can be compensated by a variation of step density along the facet, and the faceted crystal can grow in a stable manner. The convergence described above explains this phenomenon from a mathematical point of view.

\section{Appendix}

5.1. Explanation for a derivation of (1.1). In this subsection we derive the equation (1.1) from the evolution of the hypersurface $\left\{\Gamma_{t}\right\}_{t \geq 0} \subset \mathbb{R}^{N+1}$ moving according to the law of propagation

$$
V_{\varepsilon}\left(x, x_{N+1}, t\right)=\tilde{\sigma}\left(x, x_{N+1}\right) m\left(\frac{\|p\|}{\varepsilon}\right)-\tilde{f}\left(x, x_{N+1}\right) \quad \text { on } \Gamma_{t},
$$

where $\varepsilon>0, V_{\varepsilon}$ is the normal growth rate at the surface, $p$ is the step density and $\tilde{\sigma}, \tilde{f}: \mathbb{R}^{N+1} \rightarrow \mathbb{R}$ are given functions which satisfy

$$
\tilde{\sigma}(x, 0)=\sigma(x), \tilde{f}(x, 0)=f(x) \text { for all } x \in \mathbb{R}^{N} .
$$

We refer to the literatures [9, 11, 12, 14 for the background of the physical model in crystal growth. Let us consider the graph representation of the above evolution and therefore introduce the function $v^{\varepsilon}$ which satisfies $\Gamma_{t}=\left\{\left(x,-v^{\varepsilon}(x, t)\right) \mid x \in \mathbb{R}^{N}\right\}$. Then the step density and the growth rate perpendicular to the $x$-axis are expressed by the gradient of $v^{\varepsilon}$, i.e., $p=D v^{\varepsilon}(x)$ and $v_{t}^{\varepsilon}$, respectively. Thus, the above surface evolution equation can be written by

$$
v_{t}^{\varepsilon}+H_{\varepsilon}\left(x, v^{\varepsilon}, D v^{\varepsilon}\right)=0 \quad \text { in } \mathbb{R}^{N} \times(0, \infty),
$$

where

$$
H_{\varepsilon}(x, r, p):=\left(\tilde{\sigma}(x,-r) m\left(\frac{\|p\|}{\varepsilon}\right)-\tilde{f}(x,-r)\right) \sqrt{|p|^{2}+1} .
$$

We approximate $v^{\varepsilon}$ by using the microscopic time variable, i.e., $\tau=t / \varepsilon$, so that

$$
v^{\varepsilon}(x, \varepsilon \tau)=\varepsilon u(x, \tau)+o(\varepsilon) \quad \text { as } \varepsilon \rightarrow 0,
$$

where $o:[0, \infty) \rightarrow[0, \infty)$ is a function which satisfies $o(r) / r \rightarrow 0$ as $r \rightarrow 0$. By replacing $\tau$ by $t$, we see that formally $u$ solves (C). We present a rigorous argument in

Proposition 5.1. Assume that $u_{0}$ and $\tilde{\sigma}, \tilde{f}$ are bounded in $\mathbb{R}^{N}$ and $\mathbb{R}^{N+1}$, respectively. Let $v^{\varepsilon}$ be the viscosity solutions of (5.2) with the initial value $u_{0}$. Then,

$$
u^{\varepsilon}(x, t):=\frac{1}{\varepsilon} v^{\varepsilon}(x, \varepsilon t)
$$

converges to the viscosity solution of $(\mathrm{C})$ uniformly on every compact set of $\mathbb{R}^{N} \times$ $[0, \infty)$ as $\varepsilon \rightarrow 0$.

The idea of the proof is essentially the same as in [44, where it is assumed that $\tilde{\sigma}=\sigma, f=0$ with $N=1$.

Proof. It is easily seen that $u^{\varepsilon}$ satisfies

$$
\begin{cases}u_{t}^{\varepsilon}+\tilde{H}_{\varepsilon}\left(x, u^{\varepsilon}, D u^{\varepsilon}\right)=0 & \text { in } \mathbb{R}^{N} \times(0, \infty), \\ u^{\varepsilon}(x, 0)=u_{0}(x) & \text { for all } x \in \mathbb{R}^{N},\end{cases}
$$

where

$$
\tilde{H}_{\varepsilon}(x, p):=(\tilde{\sigma}(x,-\varepsilon r) m(\|p\|)-\tilde{f}(x,-\varepsilon r)) \sqrt{\varepsilon|p|^{2}+1} .
$$


Define the functions $u^{-}, u^{+}: \mathbb{R}^{N} \times(0, \infty) \rightarrow \mathbb{R}$ by

$$
\begin{aligned}
& u^{-}(x, t):=-\sup _{x \in \mathbb{R}^{N}, r \in \mathbb{R}}\left|\tilde{\sigma}(x, r) m_{0}-\tilde{f}(x, r)\right| t-C, \\
& u^{+}(x, t):=\sup _{x \in \mathbb{R}^{N}, r \in \mathbb{R}}\left|\tilde{\sigma}(x, r) m_{0}-\tilde{f}(x, r)\right| t+C,
\end{aligned}
$$

where $C>0$ is the constant with $C \geq \sup \left|u_{0}\right|$. Then it is easy to check that $u^{-}$, $u^{+}$are the viscosity subsolution and the viscosity supersolution of (5.3) with the initial value $u_{0}$, respectively.

By a comparison principle for (5.3) we have

$$
u^{-} \leq u_{\varepsilon} \leq u^{+} \quad \text { on } \mathbb{R}^{N} \times(0, \infty)
$$

If one takes half relaxed limits

$$
\begin{aligned}
& \bar{u}(x, t):=\limsup _{t \rightarrow \infty}{ }^{*} u^{\varepsilon}(x, t), \\
& \underline{u}(x, t):=\liminf _{t \rightarrow \infty} u^{\varepsilon}(x, t),
\end{aligned}
$$

we observe that $\bar{u}, \underline{u}$ are the viscosity subsolution and the viscosity supersolution of (C), respectively, by the usual stability result of the viscosity solution, since $\tilde{H}_{\varepsilon}$ converges to $H(x, p)-f(x)$ locally uniformly for $(x, p) \in \mathbb{R}^{2 N}$ as $\varepsilon \rightarrow 0$. By the comparison principle for $(\mathrm{C})$ again, we have

$$
\bar{u} \leq \underline{u} \text { on } \mathbb{R}^{N} \times[0, \infty),
$$

which implies that $\bar{u}=\underline{u}$ and $u^{\varepsilon}$ converges to the viscosity solution of (C) uniformly on every compact set of $\mathbb{R}^{N} \times[0, \infty)$ as $\varepsilon \rightarrow 0$.

Theorems 4.1 and 4.7 (see also Corollary 4.8) now give a clear view of the solution $v^{\varepsilon}$ on the effective domain $\Omega_{e}$ of (1.1) with the initial value $u_{0}$. We have

$$
\begin{aligned}
v^{\varepsilon}(x, t) & =\varepsilon u\left(x, \frac{t}{\varepsilon}\right)+o(\varepsilon) \\
& =\varepsilon \phi_{\infty}(x)+c t+o(\varepsilon) \text { for all } x \in \Omega_{e},
\end{aligned}
$$

where $o:[0, \infty) \rightarrow[0, \infty)$ is a function which satisfies $o(r) / r \rightarrow 0$ as $r \rightarrow 0$ and $c, \phi_{\infty}$ are the constant and the function given by (4.1) and (4.10). Therefore, we see that roughly speaking, the growing facet moving according to (5.1) is flat up to order $\varepsilon$ with speed $c$ on the effective domain $\Omega_{e}$.

Remark 5.1.

(i) We can consider the case where the surface supersaturation depends on the curvature. More precisely, let us add the curvature term to (5.1) and consider

$$
V_{\varepsilon}\left(x, x_{N+1}, t\right)=\left(\sigma\left(x, x_{N+1}\right)-\operatorname{div}\left(n\left(x, x_{N+1}\right)\right)\right) m\left(\frac{\|p\|}{\varepsilon}\right)-f\left(x, x_{N+1}\right) \quad \text { on } \Gamma_{t} .
$$

This is an example of mean curvature flow equations with an anisotropic mobility and a driving force term when $f=0$ as is found for example in Section 1.5.1 of [19]. Then the microscopic time scale approximated equation of the graph represented equation of (5.4) is the same as (1.1). Indeed, let $v^{\varepsilon}$ be a viscosity solution of the graph represented equation of (5.4) and set $u^{\varepsilon}(x, t)=(1 / \varepsilon) v^{\varepsilon}(x, \varepsilon t)$. Then it is easy to check (see 19 for instance) that $u^{\varepsilon}$ satisfies

$u_{t}^{\varepsilon}+\tilde{H}_{\varepsilon}\left(x, u^{\varepsilon}, D u^{\varepsilon}\right)-m\left(\left\|D u^{\varepsilon}\right\|\right) \operatorname{div}\left(\frac{\varepsilon D u^{\varepsilon}}{\sqrt{\varepsilon|D u|^{2}+1}}\right) \sqrt{\varepsilon|D u|^{2}+1}=0$ in $\mathbb{R}^{N} \times(0, \infty)$. 
By the same argument as in the proof of Proposition [5.1 we see that $u^{\varepsilon}$ converges to a solution of (1.1) locally uniformly in $\mathbb{R}^{N} \times(0, \infty)$.

(ii) In the theory of crystal growth the place where $\sigma$ takes its maximam on a crystal surface is considered as a step source when the surface is flat. The function $f$ gives a nucleation density of the crystal. Our assumption (B2) says that there is a step source in the place where no nucleation occurs.

5.2. Another characterization of solutions of (S). In this subsection, we consider problem (S) and equation (4.8). We present the definition of solutions of (4.8) introduced in [21] and the equivalence between them.

Definition 2. Let $u$ be a function on $\mathbb{R}^{N}$ to $\mathbb{R} \cup\{ \pm \infty\}$. We call $u$ a subsolution (resp., supersolution) of (4.8) if $\bar{u}(x)<\infty$ (resp., $\underline{u}(x)>-\infty$ ) for all $x \in \mathbb{R}^{N}$ and $\bar{u}$ is a viscosity subsolution (resp., $\underline{u}$ is a viscosity supersolution). We call $u$ a solution if $u$ is a subsolution and a supersolution. Here we denote $\left(u_{-}\right)^{*}\left(\operatorname{resp} .,\left(u_{+}\right)_{*}\right)$ by $\bar{u}$ (resp., $\underline{u}$ ), where

$$
\begin{aligned}
& u_{+}(x):= \begin{cases}+\infty & \text { if } u(x)=-\infty, \\
u(x) & \text { if } u(x)>-\infty,\end{cases} \\
& u_{-}(x):= \begin{cases}-\infty & \text { if } u(x)=+\infty \\
u(x) & \text { if } u(x)<+\infty,\end{cases}
\end{aligned}
$$

and $v^{*}$ (resp., $v_{*}$ ) is the upper semicontinuous envelope (resp., the lower semicontinuous envelope) of a function $v$, i.e.,

$$
\begin{gathered}
v^{*}(x):=\lim _{r \rightarrow 0} \sup \left\{v(y)\left|y \in \mathbb{R}^{N},\right| x-y \mid \leq r\right\}, \\
\text { (resp., } \left.v_{*}(x):=\lim _{r \rightarrow 0} \inf \left\{v(y)\left|y \in \mathbb{R}^{N},\right| x-y \mid \leq r\right\}\right) .
\end{gathered}
$$

Proposition 5.2. Assume that (B1) holds. Let $u$ be a supersolution of (4.8) in the sense of Definition 2, Then we have

$$
\underline{u}(x)=\infty \quad \text { for all } x \in \Omega_{d} .
$$

Proof. Suppose that there exists $\hat{x} \in \Omega_{d}$ such that $\underline{u}(\hat{x}) \neq \infty$. Note that $\Omega_{d}$ is an open set. Since $\underline{u}$ is locally bounded from below, there exist $r>0$ and $M>0$ such that $\underline{u}(x) \geq-M$ for all $x \in B(\hat{x}, r) \subset \Omega_{d}$.

It is easily seen that

$$
\underset{\min (\hat{x}, r)}{ }\left\{\underline{u}(x)-\underline{u}(\hat{x})+\frac{1}{2 \varepsilon}|x-\hat{x}|^{2}\right\} \leq 0 .
$$

Let $x_{\varepsilon}$ be a point which gives the minimum of the above. We may assume that $x_{\varepsilon} \in B(\hat{x}, r)$ for a suitable small $\varepsilon>0$. Then we have

$$
\sigma\left(x_{\varepsilon}\right) m\left(\left\|\frac{x_{\varepsilon}-\hat{x}}{\varepsilon}\right\|\right)<\sigma\left(x_{\varepsilon}\right)<f\left(x_{\varepsilon}\right)-c,
$$

which contradicts that $u$ is a supersolution of (4.8).

Proposition 5.3. Assume that (B1) holds. Let $u: \mathbb{R}^{N} \rightarrow \mathbb{R} \cup\{ \pm \infty\}$ be a subsolution (resp., a supersolution) of (4.8) in the sense of Definition 2 . Assume that $u \in C\left(\bar{\Omega}_{e}\right)$. Then $u$ is a subsolution (resp., supersolution) of $(\mathrm{S})$ in the sense of Definition 1 . 
Proof. Since it is clear that a subsolution of (4.8) in the sense of Definition 2 is a subsolution of $(\mathrm{S})$ in the sense of Definition 1, we only prove that a supersolution of (4.8) in the sense of Definition 2 is a supersolution of $(S)$ in the sense of Definition 1.

Suppose that $u-\phi$ takes a minimum at $\hat{x} \in \partial \Omega_{e}$ for some $\phi \in C^{1}\left(\bar{\Omega}_{e}\right)$. Extend $\phi$ to the function $\tilde{\phi}$ defined on $\mathbb{R}^{N}$ such that $\tilde{\phi} \in C^{1}\left(\mathbb{R}^{N}\right)$ and $\tilde{\phi}=\phi$ on $\bar{\Omega}_{e}$. Then $\underline{u}-\tilde{\phi}$ takes a minimum at $\hat{x} \in \partial \Omega_{e}$ by Proposition 5.2. Therefore, we have

$$
\sigma(\hat{x}) m(\|\phi(\hat{x})\|)<\sigma(\hat{x})=f(\hat{x})-c,
$$

which contradicts the fact that $u$ is a supersolution in the sense of Definition 1 .

Proposition 5.4. Assume that (B1) holds. Let $u \in C\left(\bar{\Omega}_{e}\right)$ (resp., $v \in C\left(\bar{\Omega}_{e}\right)$ ) be a solution in the sense of Definition 1 . Extend $u$ to the function on $\mathbb{R}^{N}$ such that $\tilde{u}=u$ on $\bar{\Omega}_{e}$ and $\tilde{u}(x)=\infty$ for all $x \in \mathbb{R}^{N} \backslash \bar{\Omega}_{e}$. Then $\tilde{u}$ is a subsolution (resp., supersolution) of (4.8) in the sense of Definition 1 .

Proof. By the definition of a supersolution we have $D^{-}(\tilde{u})(x)=D^{-} \tilde{u}(x)=\emptyset$ for all $x \in \partial \Omega_{e}$. Thus, it is clear that $u$ is a supersolution of (4.8) in the sense of Definition 2 ,

For any $p \in D^{+} \overline{(\tilde{u})}(x)=D^{+} \tilde{u}(x)$ and $x \in \partial \Omega_{e}$, we have

$$
\sigma(x) m(\|p\|)<\sigma(x)=f(x)-c,
$$

which implies that $u$ is a subsolution of (4.8) in the sense of Definition 2

\subsection{Uniform continuity of subsolutions for (N).}

Proof of Proposition 3.6. We fix any $z \in \partial \Omega$, and by (A8) choose $r>0$ and $b \in$ $C\left(\mathbb{R}^{n-1}\right)$ so that, after relabelling and re-orienting the coordinates if necessary, we have

$$
\Omega \cap B(z, r)=\left\{\left(x^{\prime}, x_{n}\right) \in \mathbb{R}^{n} \mid x_{n}>b\left(x^{\prime}\right)\right\} \cap B(z, r) .
$$

Here and henceforth, we write $x=\left(x^{\prime}, x_{n}\right)$ for $x \in \mathbb{R}^{n}$, where $x^{\prime} \in \mathbb{R}^{n-1}$. Let $m_{z}$ be the modulus of continuity of $b$ on the ball $\bar{B}\left(z^{\prime}, r\right) \subset \mathbb{R}^{n-1}$. We choose a $\delta \in(0, r / 2)$ so that $m_{z}(\delta)<r / 4$.

Let $x, y \in \Omega \cap B(z, \delta / 2)$, and set $\varepsilon:=|x-y|, \xi:=\left(x^{\prime}, x_{n}+m_{z}(\varepsilon)\right)$, and $\eta:=\left(y^{\prime}, y_{n}+m_{z}(\varepsilon)\right)$. Consider the line segments $[x, \xi],[y, \eta]$ and $[\xi, \eta]$. Noting that $\xi, \eta \in B(z, r / 2)$, we easily see that $[x, \xi] \cup[y, \eta] \subset \Omega$ and that $[\xi, \eta] \subset B(z, r / 2)$. Observe that for any $t \in[0,1]$,

$$
\begin{aligned}
& b\left(t x^{\prime}+(1-t) y^{\prime}\right) \\
& \leq \min \left\{b\left(x^{\prime}\right)+m_{z}(\varepsilon), b\left(y^{\prime}\right)+m_{z}(\varepsilon)\right\} \\
& <\min \left\{x_{n}, y_{n}\right\}+m_{z}(\varepsilon) \leq t\left(x_{n}+m_{z}(\varepsilon)\right)+(1-t)\left(y_{n}+m_{z}(\varepsilon)\right),
\end{aligned}
$$

which reads $b\left(t \xi^{\prime}+(1-t) \eta^{\prime}\right)<t \xi_{n}+(1-t) \eta_{n}$. We thus conclude that

$$
[x, \xi] \cup[\xi, \eta] \cup[y, \eta] \subset \Omega .
$$

By [29, Proposition 2.1] there exist $\delta, C>0$ such that

$$
L(x, p) \leq C \quad \text { for all }(x, p) \in \bar{\Omega} \times B(0, \rho) .
$$

Set

$$
t_{1}=t_{3}=\frac{m_{z}(\varepsilon)}{\rho}, t_{2}=\frac{\varepsilon}{\rho} \text { and } t=t_{1}+t_{2}+t_{3} .
$$


Define the trajectories $\gamma_{i}:\left[0, t_{i}\right] \rightarrow \Omega$ and $\gamma:[0, t] \rightarrow \Omega$ by

$$
\gamma_{1}(s)=y+\frac{\rho(\eta-y)}{m_{z}(\varepsilon)} s, \gamma_{2}(s)=\eta+\frac{\rho(\xi-\eta)}{\varepsilon} s, \gamma_{3}(s)=\xi+\frac{\rho(x-\xi)}{m_{z}(\varepsilon)} s
$$

and

$$
\gamma(s):= \begin{cases}\gamma_{1}(s) & \text { for all } s \in\left[0, t_{1}\right] \\ \gamma_{2}\left(s-t_{1}\right) & \text { for all } s \in\left[t_{1}, t_{1}+t_{2}\right] \\ \gamma_{3}\left(s-t_{1}-t_{2}\right) & \text { for all } s \in\left[t_{1}+t_{2}, t\right] .\end{cases}
$$

Then, we have $\gamma \in \mathcal{C}(x, t ; y, 0)$.

By (A7) we calculate that

$$
\begin{aligned}
d(x, y) \leq & \int_{0}^{t} L(\gamma(s), \dot{\gamma}(s))+h(\gamma(s)) d s \\
\leq & C t+\int_{0}^{t} h(\gamma(s)) d s \\
= & C t+\int_{0}^{t_{1}} h\left(\gamma_{1}(s)\right) d s+\int_{0}^{t_{2}} h\left(\gamma_{2}(s)\right) d s+\int_{0}^{t_{3}} h\left(\gamma_{3}(s)\right) d s \\
= & C t+t_{1} \int_{0}^{1} h(y+r(\eta-y)) d r+t_{2} \int_{0}^{1} h(\eta+r(\xi-\eta)) d r \\
& +t_{3} \int_{0}^{1} h(\xi+r(x-\xi)) d r \\
= & C t+\frac{1}{\rho}\left[\int_{0}^{1} h(y+r(\eta-y))|\eta-y| d r+\int_{0}^{1} h(\eta+r(\xi-\eta))|\xi-\eta| d r\right. \\
& \left.+\int_{0}^{1} h(\xi+r(x-\xi))|x-\xi| d r\right] \\
\leq & \frac{C}{\rho}\left(\varepsilon+2 m_{z}(\varepsilon)\right)+\frac{1}{\rho}\left(\omega(\varepsilon)+2 \omega\left(m_{z}(\varepsilon)\right)\right) .
\end{aligned}
$$

Setting $\omega_{z}(\varepsilon):=\frac{C}{\rho}\left(\varepsilon+2 m_{z}(\varepsilon)\right)+\frac{1}{\rho}\left(\omega(\varepsilon)+2 \omega\left(m_{z}(\varepsilon)\right)\right.$, we have

$$
d(x, y) \leq \omega_{z}(\varepsilon)
$$

By the standard compactness argument, we find an open neighborhood $V$ of $\partial \Omega$, relative to $\Omega$, and a modulus $\omega_{0}$ such that $d(x, y) \leq \omega_{0}(|x-y|)$ for all $x, y \in V$. We choose a compact neighborhood $W \subset \Omega$ of $\Omega \backslash V$ and observe that $u$ is Lipschitz continuous on $W \times W$. Note that

$$
d(y, y)=0 \text { and } d\left(x_{1}, y\right)-d\left(x_{2}, y\right) \leq d\left(x_{1}, x_{2}\right)
$$

for all $x_{1}, x_{2}, y \in \Omega$. It is now easy to conclude that $d$ is uniformly continuous on $\Omega \times \Omega$.

Lemma 3.3 is a straightforward result of Proposition 3.6 .

5.4. Lipschitz estimates and derivative bounds. We consider the Lipschitz constant of viscosity solutions of

$$
\|D u(x)\| \leq C \text { in } \Omega,
$$

where $C$ is a positive constant and $\Omega$ is a domain in $\mathbb{R}^{N}$. 
Proposition 5.5. If $|u(x)-u(y)| \leq C\|x-y\|_{*}$ for all $x, y \in \Omega$, then $\|D u\| \leq C$ in $\Omega$ in the viscosity sense.

Proof. Note that every norm in a finite-dimensional space is equivalent. In view of Rademacher's theorem, for almost every $y \in \Omega$, there exists $p_{y} \in \mathbb{R}^{N}$ such that

$$
\frac{\left|u(x)-u(y)-p_{y} \cdot(x-y)\right|}{\|x-y\|_{*}}=o(1) \quad \text { as } y \rightarrow x,
$$

where $o(1) \rightarrow 0$ as $y \rightarrow x$.

Choose $q_{y} \in \mathbb{R}^{N} \backslash\{0\}$ such that $\left\|p_{y}\right\|=\left|p_{y} \cdot q_{y}\right| /\left\|q_{y}\right\|_{*}$ and set $x_{\varepsilon}:=y+\varepsilon q_{y} /\left\|q_{y}\right\|_{*}$ for $\varepsilon>0$. Then we have

$$
\begin{aligned}
\left\|p_{y}\right\| & =\left|\frac{u\left(x_{\varepsilon}\right)-u(y)-p_{y} \cdot\left(x_{\varepsilon}-y\right)}{\left\|x_{\varepsilon}-y\right\|_{*}}-\frac{u\left(x_{\varepsilon}\right)-u(y)}{\left\|x_{\varepsilon}-y\right\|_{*}}\right| \\
& \leq\left|\frac{u\left(x_{\varepsilon}\right)-u(y)-p_{y} \cdot\left(x_{\varepsilon}-y\right)}{\left\|x_{\varepsilon}-y\right\|_{*}}\right|+C \\
& \rightarrow C \quad \text { as } \varepsilon \rightarrow 0 .
\end{aligned}
$$

Therefore, we have

$$
\|D u(x)\| \leq C \text { for almost every } x \in \Omega .
$$

Since the map $p \mapsto\|p\|$ is convex, we have

$$
\|D u\| \leq C \text { in } \Omega \text { in the viscosity sense. }
$$

Lemma 5.6. Let $u$ be a viscosity solution of (5.5). For any $y \in \Omega$ and $r>0$ such that $B(y, r) \subset \Omega$, we have

$$
|u(x)-u(y)| \leq C\|x-y\|_{*} \quad \text { for all } x \in B\left(y, \frac{r}{4}\right) .
$$

In order to prove Lemma 5.6 we prepare the following lemma.

Lemma 5.7. Let $\|\cdot\|_{*}$ be the dual norm of $\|\cdot\|$ and $a>0$. Set $\psi_{a}(x):=\rho_{a} *\|\cdot\|_{*}(x)$, where $\rho_{a}$ is a standard mollification kernel and define the function $\tilde{\psi}_{a}$ by $\tilde{\psi}_{a}(x):=$ $\psi_{a}(x /|x|)|x|$. Then $\tilde{\psi}_{a} \in C^{1}\left(\mathbb{R}^{n} \backslash\{0\}\right)$ is homogeneous with degree 1 and satisfies

$$
\left\|D \tilde{\psi}_{a}\right\| \geq 1-\frac{C a}{\|x\|_{*}} \text { for all } x \in \mathbb{R}^{n} \backslash\{0\} \text { and some } C>0 .
$$

Proof. It is clear to see that $\tilde{\psi}_{a} \in C^{1}\left(\mathbb{R}^{n} \backslash\{0\}\right)$ is homogeneous with degree 1 . By the duality of norm and the Euler homogeneous function theorem we have

$$
\left\|D \tilde{\psi}_{a}(x)\right\|=\sup _{\|y\|_{*} \leq 1}\left|D \tilde{\psi}_{a} \cdot y\right| \geq\left|D \tilde{\psi}_{a} \cdot \frac{x}{\|x\|_{*}}\right|=\frac{\tilde{\psi}_{a}(x)}{\|x\|_{*}} \geq 1-\frac{C a}{\|x\|_{*}}
$$

for some $C>0$.

Proof of Lemma 5.6. Let $\varepsilon>0$ and consider a function $\phi \in C^{1}(B(y, r / 2) \backslash\{y\}) \cap$ $C^{0}(B(y, r / 2))$ which satisfies

$$
\begin{aligned}
& \phi(x)=u(y)+(C+\varepsilon) \tilde{\psi}_{a}(x-y) \quad \text { for all } x \in B\left(y, \frac{r}{4}\right), \\
& \|D \phi(x)\| \geq C+\varepsilon \text { in } B\left(y, \frac{r}{2}\right) \backslash \bar{B}\left(y, \frac{r}{4}\right) \text { and } \\
& u(x)<\phi(x) \text { for all } x \in \partial B\left(y, \frac{r}{2}\right),
\end{aligned}
$$


where $\tilde{\psi}_{a}$ is the function defined in Lemma 5.7. Let $z \in \bar{B}\left(y, \frac{r}{2}\right)$ be a point such that $\max _{\bar{B}\left(y, \frac{r}{2}\right)}(u-\phi)=(u-\phi)(z)$. Noting that $\max _{\bar{B}\left(y, \frac{r}{2}\right)}(u-\phi) \geq 0$, we see $z \in B\left(y, \frac{r}{2}\right)$. Suppose that $z \in B\left(y, \frac{r}{2}\right) \backslash\{y\}$. Then by the definition of a viscosity subsolution, we have

$$
\|D \phi(z)\| \leq C .
$$

Meanwhile, by Lemma 5.7 we have $\|D \phi(x)\| \geq(C+\varepsilon)\left(1-C a /\|x-y\|_{*}\right)$ for all $x \in B\left(y, \frac{r}{2}\right) \backslash\{y\}$. Sending $a \rightarrow 0$ yields the contradiction. Therefore, $z$ needs to be $y$. Consequently,

$$
(u-\phi)(x) \leq(u-\phi)(y)=0 \quad \text { for all } x \in B\left(y, \frac{r}{4}\right),
$$

which implies that

$$
u(x)-u(y) \leq(C+\varepsilon) \tilde{\psi}_{a}(x-y) \leq(C+\varepsilon)\left(\|x-y\|_{*}+C a\right)
$$

for all $x \in B\left(y, \frac{r}{4}\right)$. Sending $a \rightarrow 0, \varepsilon \rightarrow 0$, we get the conclusion.

Proposition 5.8. Assume that $\Omega$ is convex and let $u$ be a viscosity solution of (5.5). Then we have

$$
|u(x)-u(y)| \leq C\|x-y\|_{*} \quad \text { for any } x, y \in \bar{\Omega} .
$$

Remark 5.2. We remark that if we assume that $\Omega$ is convex, then the Lipschitz constant of solutions of (5.5) coincides with the constant in (5.5).

Proof. Fix $x, y \in \Omega$. In view of the convexity of $\Omega$, we have $[x, y] \subset \Omega$. Choose $r>0$ such that $\bigcup_{z \in[x, y]} B(z, r) \subset \Omega$. There exist $m \in \mathbb{N}$ and $\left\{\xi_{i}\right\}_{i=1, \ldots, m} \subset[x, y]$ such that $\xi_{1}=x, \xi_{m}=y, \xi_{i} \in B\left(\xi_{i+1}, r / 4\right)$ for all $i=1, \ldots, m-1$ and $|x-y|=$ $\sum_{i=1}^{m-1}\left|\xi_{i}-\xi_{i+1}\right|$. By Lemma [5.6 we have

$$
|u(x)-u(y)| \leq \sum_{i=1}^{m-1}\left|u\left(\xi_{i}\right)-u\left(\xi_{i+1}\right)\right| \leq \sum_{i=1}^{m-1} C\left\|\xi_{i}-\xi_{i+1}\right\|_{*}=C\|x-y\|_{*} .
$$

Fix $x, y \in \partial \Omega$. Choose $\left\{x_{j}\right\}_{j \in \mathbb{N}},\left\{y_{j}\right\}_{j \in \mathbb{N}}$ such that $x_{j} \rightarrow x$ and $y_{j} \rightarrow y$ as $j \rightarrow \infty$. We have $\left|u\left(x_{j}\right)-u\left(y_{j}\right)\right| \leq C\left\|x_{j}-y_{j}\right\|_{*}$. Sending $j \rightarrow \infty$, we get $|u(x)-u(y)| \leq$ $C\|x-y\|_{*}$.

\section{ACKNOWLEDGEMENTS}

The authors are grateful to Professor Etsuro Yokoyama for his valuable comments on models. The third author is grateful to Professor Hitoshi Ishii for reminding them of the work 34 at the beginning of this study and to Professor Guy Barles for his encouragement. The authors thank the anonymous referee for a careful reading of the previous version of the manuscript and valuable comments. This work was completed while the authors visited the Department of Mathematics and the Research Center for Integrative Mathematics, Hokkaido University for a minisemester on evolution of interfaces, Sapporo 2010. Its hospitality is gratefully acknowledged. This work was partially done while the third author visited the 
Laboratoire de Mathématiques et Physique Théorique, Université de Tours, and Mathematics Department, University of California, Berkeley. He is grateful for their hospitality.

\section{REFERENCES}

1. M. Bardi, and I. Capuzzo-Dolcetta, Optimal control and viscosity solutions of HamiltonJacobi-Bellman equations, With appendices by Maurizio Falcone and Pierpaolo Soravia. Systems \& Control: Foundations \& Applications. Birkhäuser Boston, Inc., Boston, MA, 1997. MR.1484411(99e:49001)

2. G. Barles, F. Da Lio, On the generalized Dirichlet problem for viscous Hamilton-Jacobi equations, J. Math. Pures Appl. (9) 83 (2004), no. 1, 53-75. MR2023054 (2005e:35107)

3. G. Barles, H. Ishii and H. Mitake, On the large time behavior of solutions of Hamilton-Jacobi equations associated with nonlinear boundary conditions, Arch. Ration. Mech. Anal. 204 (2012), 515-558.

4. G. Barles and H. Mitake, A PDE approach to large-time asymptotics for boundary-value problems for nonconvex Hamilton-Jacobi equations, Comm. Partial Differential Equations 37 (2012), 136-168. MR2864810

5. G. Barles and B. Perthame, Discontinuous solutions of deterministic optimal stopping time problems, RAIRO Model. Math. Anal. Numer. 21 (1987), no. 4, 557-579. MR921827 (88k:49032)

6. G. Barles and J.-M. Roquejoffre, Ergodic type problems and large time behaviour of unbounded solutions of Hamilton-Jacobi equations, Comm. Partial Differential Equations 31 (2006), no. 7-9, 1209-1225. MR 2254612 (2007f:35023)

7. G. Barles and P. E. Souganidis, On the large time behavior of solutions of Hamilton-Jacobi equations, SIAM J. Math. Anal. 31 (2000), no. 4, 925-939. MR1752423 (2002b:49056)

8. E. N. Barron and R. Jensen, Semicontinuous viscosity solutions for Hamilton-Jacobi equations with convex Hamiltonians, Comm. Partial Differential Equations 15 (1990), no. 12, 1713-1742. MR.1080619 (91h:35069)

9. W. K. Burton, N. Cabrera, F. C. Frank, The growth of crystals and the equilibrium structure of their surfaces, Philos. Trans. Roy. Soc. London. Ser. A. 243 (1951), 299-358. MR0043005 (13:196f)

10. I. Capuzzo Dolcetta and P.-L. Lions, Hamilton-Jacobi equations with state constraints, Trans. Amer. Math. Soc. 318 (1990), no. 2, 643-683. MR951880 (90g:49021)

11. A. A. Chernov, Application of the method of characteristics to the theory of the growth from of crystals, Soviet Phys. - Crystal.8 (1964), 401-405.

12. A. A. Chernov, Stability of faceted shapes, J. Crystal Growth 24/25 (1974), 11-31.

13. A. Davini and A. Siconolfi, A generalized dynamical approach to the large time behavior of solutions of Hamilton-Jacobi equations, SIAM J. Math. Anal. 38 (2006), no. 2, 478-502 MR2237158 (2007d:49047)

14. W. E, N. K. Yip, Continuum theory of epitaxial crystal growth. I, J. Statistical Physics, 104, (2001), 221-253. MR1925170 (2003g:82070)

15. L. C. Evans, R. F. Gariepy, Measure theory and fine properties of functions, Studies in Advanced Mathematics, CRC Press, Boca Raton, FL, 1992. MR1158660 (93f:28001)

16. A. Fathi, Théorème KAM faible et théorie de Mather sur les systèmes lagrangiens, C. R. Acad. Sci. Paris Sér. I Math. 324 (1997), no. 9, 1043-1046. MR.1451248 (98g:58151)

17. A. Fathi, Sur la convergence du semi-groupe de Lax-Oleinik, C. R. Acad. Sci. Paris Sér. I Math. 327 (1998), no. 3, 267-270. MR1650261 (2000a:37058)

18. A. Fathi and A. Siconolfi, PDE aspects of Aubry-Mather theory for quasiconvex Hamiltonians, Calc. Var. Partial Differential Equations 22 (2005), no. 2, 185-228. MR2106767|(2006f:35023)

19. Y. Giga, Surface evolution equations. A level set approach, Monographs in Mathematics, 99. Birkhäuser Verlag, Basel, 2006. MR2238463(2007j:53071)

20. Y. Giga and N. Hamamuki, Hamilton-Jacobi equations with discontinuous source terms, Comm. Partial Differential Equations 38 (2013), no. 2, 199-243. MR.3009078 
21. Y. Giga, Q. Liu and H. Mitake, Large-time asymptotics for one-dimensional Dirichlet problems for Hamilton-Jacobi equations with noncoercive Hamiltonians, J. Differential Equations, 252, (2012), 1263-1282. MR2853538

22. N. Hamamuki, On large time behavior of Hamilton-Jacobi equations with discontinuous source terms, preprint.

23. D. Margetis and R. V. Kohn, Continuum relaxation of interacting steps on crystal surfaces in 2+1 dimensions, Multiscale Model. Simul. 5 (2006), 729-758. MR2257233 (2007i:74008)

24. N. Ichihara and H. Ishii, Asymptotic solutions of Hamilton-Jacobi equations with semiperiodic Hamiltonians, Comm. Partial Differential Equations 33 (2008), no. 4-6, 784-807. MR2424378 (2009h:35047)

25. N. Ichihara and H. Ishii, The large-time behavior of solutions of Hamilton-Jacobi equations on the real line, Methods Appl. Anal. 15, No.2, pp.223-242 (2008). MR2481681|(2010e:35051)

26. N. Ichihara and H. Ishii, Long-time behavior of solutions of Hamilton-Jacobi equations with convex and coercive Hamiltonians, Arch. Ration. Mech. Anal. 194 (2009), no. 2, 383-419. MR2563634 (2011b:35067)

27. H. Ishii, Uniqueness of unbounded viscosity solution of Hamilton-Jacobi equations, Indiana Univ. Math. J. 33 (1984), no. 5, 721-748. MR756156 (85h:35057)

28. H. Ishii, Perron's method for Hamilton-Jacobi equations, Duke Math. J. 55 (1987), no. 2, 369-384. MR894587 (89a:35053)

29. H. Ishii, A boundary value problem of the Dirichlet type for Hamilton-Jacobi equations, Ann. Scuola Norm. Sup. Pisa Cl. Sci. (4) 16 (1989), no. 1, 105-135. MR.1056130 (91f:35071)

30. H. Ishii, Asymptotic solutions for large time of Hamilton-Jacobi equations in Euclidean $n$ space, Ann. Inst. H. Poincaré Anal. Non Linéaire, 25 (2008), no 2, 231-266. MR2396521 (2009h:35048)

31. H. Ishii, Weak KAM aspects of convex Hamilton-Jacobi equations with Neumann type boundary conditions, J. Math. Pures Appl. (9) 95(1), 99-135, 2011. MR2746439(2012d:37144)

32. H. Ishii, Long-time asymptotic solutions of convex Hamilton-Jacobi equations with Neumann type boundary conditions, Calc. Var. Partial Differ. Equ., 42, 189-209. 2011. MR2819634 (2012g:35034)

33. H. Ishii, H. Mitake, Representation formulas for solutions of Hamilton-Jacobi equations with convex Hamiltonians, Indiana Univ. Math. J. 56 (2007), no. 5, 2159-2183. MR2360607 (2008k:35049)

34. J.-M. Lasry, P.-L. Lions, Nonlinear elliptic equations with singular boundary conditions and stochastic control with state constraints. I. The model problem, Math. Ann. 283 (1989), no. 4, 583-630. MR.990591 (90f:35072)

35. P.-L. Lions, Neumann type boundary conditions for Hamilton-Jacobi equations, Duke Math. J. 52 (1985), no. 4, 793-820. MR816386 (87h:35055)

36. H. Mitake, Asymptotic solutions of Hamilton-Jacobi equations with state constraints, Appl. Math. Optim. 58 (2008), no. 3, 393-410. MR2456853 (2009m:35043)

37. H. Mitake, The large-time behavior of solutions of the Cauchy-Dirichlet problem for HamiltonJacobi equations, NoDEA Nonlinear Differential Equations App. 15 (2008), no. 3, 347-362. MR2458643(2010m:35060)

38. H. Mitake, Large time behavior of solutions of Hamilton-Jacobi equations with periodic boundary data, Nonlinear Anal. 71 (2009), no. 11, 5392-5405. MR2560209 (2010m:35061)

39. G. Namah and J.-M. Roquejoffre, Remarks on the long time behaviour of the solutions of Hamilton-Jacobi equations, Comm. Partial Differential Equations 24 (1999), no. 5-6, 883893. MR1680905 (2000j:35034)

40. P. Quittner and P. Souplet, Superlinear parabolic problems, blow-up, global existence and steady states, Birkhauser (2007). MR2346798 (2008f:35001)

41. J.-M. Roquejoffre, Convergence to steady states or periodic solutions in a class of HamiltonJacobi equations, J. Math. Pures Appl. (9) 80 (2001), no. 1, 85-104. MR.1810510 (2001k:35053)

42. H. M. Soner, Optimal control with state-space constraint, I. SIAM J. Control Optim. 24 (1986), no. 3, 552-561. MR838056 (87e:49029)

43. P. Souplet, Q. S. Zhang, Global solutions of inhomogeneous Hamilton-Jacobi equations, J. Anal. Math. 99 (2006), 355-396. MR2279557 (2007k:35243) 
44. E. Yokoyama, Y. Giga and P. Rybka, A microscopic time scale approximation to the behavior of the local slope on the faceted surface under a nonuniformity in supersaturation, Phys. D 237 (2008), no. 22, 2845-2855. MR2514066 (2010j:82109)

Graduate School of Mathematical Sciences, University of Tokyo, 3-8-1 Komaba, Meguro-ku, Tokyo, 153-8914, Japan - and - Department of Mathematics, Faculty of Sciences, King Abdulaziz University, P.O. Box 80203, Jeddah 21589, Saudi Arabia

E-mail address: labgiga@ms.u-tokyo.ac.jp

Graduate School of Mathematical Sciences, University of Tokyo, 3-8-1 Komaba, MEGURO-KU, TOKYO, 153-8914, JAPAN

Current address: Department of Mathematics, University of Pittsburgh, Pittsburgh, Pennsylvania 15260

E-mail address: qingliu@pitt.edu

Department of Applied Mathematics, Graduate School of Engineering, Hiroshima University, 1-4-1 Kagamiyama, Higashi-Hiroshima-Shi 739-8527, JAPAN

Current address: Department of Applied Mathematics, Faculty of Science, Fukuoka University, Fukuoka 814-0180, Japan

E-mail address: mitake@math.sci.fukuoka-u.ac.jp 